\title{
Binary evolution using the theory of osculating orbits
}

\section{Conservative Algol evolution ${ }^{\star}$}

\author{
P. J. Davis ${ }^{1}$, L. Siess ${ }^{1}$, and R. Deschamps ${ }^{1,2}$ \\ ${ }^{1}$ Institut d'Astronomie et d'Astrophysique (IAA), Université Libre de Bruxelles (ULB), CP226, boulevard du Triomphe, \\ 1050 Brussels, Belgium \\ e-mail: pdavis@ulb.ac.be \\ 2 ESO Vitacura, avenue Alonso de Córdova 3107, Vitacura, Casilla 19001, Santiago de Chile, Chile
}

Received 28 February 2014 / Accepted 20 August 2014

\begin{abstract}
Context. Studies of conservative mass transfer in interacting binary systems widely assume that orbital angular momentum is conserved. However, this only holds under physically unrealistic assumptions.

Aims. Our aim is to calculate the evolution of Algol binaries within the framework of the osculating orbital theory, which considers the perturbing forces acting on the orbit of each star arising from mass exchange via Roche lobe overflow. The scheme is compared to results calculated from a "classical" prescription.

Methods. Using our stellar binary evolution code BINSTAR, we calculate the orbital evolution of Algol binaries undergoing case A and case B mass transfer, by applying the osculating scheme. The velocities of the ejected and accreted material are evaluated by solving the restricted three-body equations of motion, within the ballistic approximation. This allows us to determine the change of linear momentum of each star, and the gravitational force applied by the mass transfer stream. Torques applied on the stellar spins by tides and mass transfer are also considered.

Results. Using the osculating formalism gives shorter post-mass transfer orbital periods typically by a factor of 4 compared to the classical scheme, owing to the gravitational force applied onto the stars by the mass transfer stream. Additionally, during the rapid phase of mass exchange, the donor star is spun down on a timescale shorter than the tidal synchronization timescale, leading to sub-synchronous rotation. Consequently, between 15 and 20 per cent of the material leaving the inner-Lagrangian point is accreted back onto the donor (so-called "self-accretion"), further enhancing orbital shrinkage. Self-accretion, and the sink of orbital angular momentum which mass transfer provides, may potentially lead to more contact binaries. Even though Algols are mainly considered, the osculating prescription is applicable to all types of interacting binaries, including those with eccentric orbits.
\end{abstract}

Key words. binaries: general - stars: evolution - stars: rotation - stars: mass-loss - celestial mechanics

\section{Introduction}

Roche lobe overflow (RLOF) gives rise to a wide variety of phenomena, with implications for many areas of astrophysics. For instance, accretion onto a massive white dwarf may lead to Type Ia supernovae (Hoyle \& Fowler 1960; Wang \& Han 2012, for a review), or X-ray emission from accreting neutron stars and black holes (e.g. Zeldovich \& Guseynov 1966). Additionally, RLOF is a viable formation channel for blue stragglers (McCrea 1964; Geller \& Mathieu 2011; Leigh et al. 2013), sub-dwarf B stars (Mengel et al. 1976; Han et al. 2002; Chen et al. 2013), and for interacting compact binaries via the common envelope phase, which is triggered by dynamically unstable RLOF from a giant or asymptotic giant branch star (Paczynski 1976; Webbink 2008).

The exchange of mass and angular momentum during RLOF causes the orbital separation to change, dictating the subsequent fate of the binary system. For a primary (i.e. the initially more massive) star of mass $M_{1}$ and a secondary star of mass $M_{2}$, the rate of change of the semi-major axis, $\dot{a}$, is determined from the rates of change of the orbital angular momentum, $\dot{J}_{\text {orb }}$, the

\footnotetext{
* Appendices are available in electronic form at http://www . aanda.org
}

primary mass, $\dot{M}_{1}$, the secondary mass, $\dot{M}_{2}$, and of the eccentricity, $\dot{e}$, according to

$\frac{\dot{a}}{a}=2 \frac{\dot{J}_{\mathrm{orb}}}{J_{\mathrm{orb}}}-2 \frac{\dot{M}_{1}}{M_{1}}-2 \frac{\dot{M}_{2}}{M_{2}}+\frac{\dot{M}_{1}+\dot{M}_{2}}{M_{1}+M_{2}}+\frac{2 e \dot{e}}{1-e^{2}}$

For conservative mass transfer within circular orbits $(e=0)$, it is assumed that mass and orbital angular momentum are conserved, i.e. $\dot{M}_{2}=-\dot{M}_{1}$, and $\dot{J}_{\text {orb }}=0$ (e.g. Paczyński 1971). Here, mass transfer from the more massive primary to the less massive secondary $\left(q=M_{1} / M_{2}>1\right)$ leads to orbital shrinkage $(\dot{a}<0)$, while the reverse occurs for $q<1$ (see, e.g. Pringle \& Wade 1985). However, even if the mass remains in the system, orbital angular momentum may not be conserved $\left(\dot{J}_{\text {orb }} \neq 0\right)$ because of the exchange of angular momentum between the orbit and the stellar spins via tidal torques and mass transfer (Gokhale et al. 2007; Deschamps et al. 2013).

In the 60s, several works from Piotrowski (1964), Kruszewski (1964b) and Hadjidemetriou (1969a,b) evaluated the gravitational force between the material leaving the innerLagrangian, $\mathcal{L}_{1}$, point (the matter stream) and each star. As a particle travels, it generates a time-varying torque on the stars, allowing for angular momentum to be exchanged between the transferred material and the orbit. Subsequently, 
Luk'yanov (2008) demonstrated that orbital angular momentum is conserved only if the stars are point masses, if the gravitational force between the matter stream and the stars is neglected and if the velocity of the ejected (accreted) material is equal in magnitude but in the opposite direction to the orbital velocity of the mass loser (gainer; see Appendix B). In reality, the velocity of the accreted particle is determined by the initial ejection velocity, which in turn depends on the thermal sound speed in the primary's atmosphere and its rotation rate (Kruszewski 1964a; Flannery 1975).

It is widely assumed that tides enforce the synchronous rotation of the primary with the orbit during RLOF. However, there is observational evidence for super- and sub-synchronously rotating stars in circular binaries (e.g. Habets \& Zwaan 1989; Andersen et al. 1990; Meibom et al. 2006; Yakut et al. 2007). Furthermore, Pratt \& Strittmatter (1976) and Savonije (1978) argued that if the ejected material removes angular momentum faster than tides can act to synchronize the rotation, the primary star will rotate sub-synchronously and its Roche lobe radius will be affected (e.g. Limber 1963; Sepinsky et al. 2007a).

A sub-synchronously rotating primary may cause the ejected material to be accreted back onto the primary (henceforth termed "self-accretion"), which causes the orbit to shrink even when $q<1$ (Sepinsky et al. 2010). Super-synchronous rotation, on the other hand, may have the opposite effect (Kruszewski 1964b; Piotrowski 1964, 1967).

In this investigation, we apply the scheme of Hadjidemetriou (1969a,b), who derived the equations of motion of a binary system using the theory of osculating orbital elements. His scheme accounts for the transfer of linear momentum between the stars, and perturbations to the orbit due to the gravitational attraction between the stars and the mass transfer stream. Using our binary stellar evolution code BINSTAR, we calculate the resulting evolution of Algol binaries for a range of initial periods and masses. Currently, we assume that none of the exchanged mass leaves the system. Torques applied onto each star by tides and mass transfer are also included.

The paper is organized as follows. In Sect. 2 we introduce the formalism of Hadjidemetriou (1969a,b). Our results are presented in Sect. 3, and discussed in Sect. 4. We summarise and conclude our investigations in Sect. 5.

\section{Computational method}

BINSTAR is an extension of the single-star evolution code STAREVOL. Details on the stellar input physics can be found in Siess (2010), and references therein, while the binary input physics is described in Siess et al. (2013) and Deschamps et al. (2013). In this section we present our new implementation of the osculating scheme.

\subsection{Variation of the orbital parameters}

Consider a binary system with an eccentricity $e$. The stars orbit about their common centre of mass $O$ (see Fig. 1) with an orbital angular speed, $\omega$, and orbital period $P_{\text {orb }}$.

Material is ejected from the primary star at a rate $\dot{M}_{1}$ with a velocity $V_{1}$ relative to the primary's mass centre, given by

$V_{1}=W_{1}-v_{1}$

where $\boldsymbol{W}_{1}$ is the absolute velocity of the ejected material, and $\boldsymbol{v}_{1}$ is the orbital velocity of the primary's mass centre. Similarly,

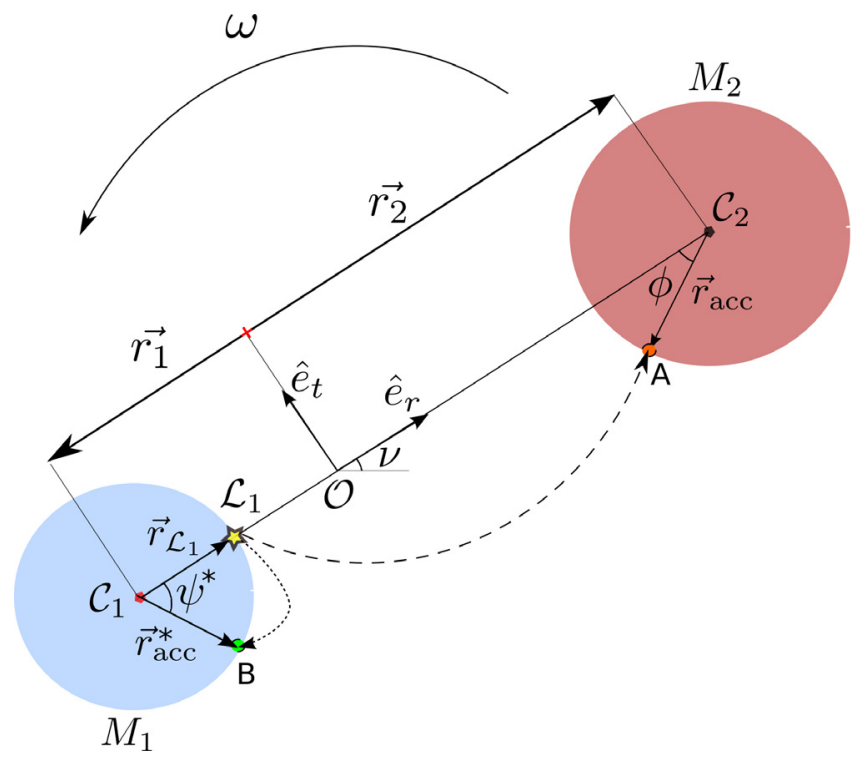

Fig. 1. Schematic of a binary system, consisting of a primary star of mass $M_{1}$, and a secondary of mass $M_{2}$ orbiting with an angular speed $\omega$, where the centre of mass of the binary system is located at $O$. The primary and secondary are respectively located at $\boldsymbol{r}_{1}$ and $\boldsymbol{r}_{2}$ with respect to $O$. Mass is ejected from the inner-Lagrangian, $\mathcal{L}_{1}$, point (yellow star), located at $\boldsymbol{r}_{\mathcal{L}_{1}}$ with respect to the primary's mass centre, $\mathcal{C}_{1}$. Material falls towards the secondary (dashed line) and is accreted onto its surface (or at the edge of an accretion disc) at $A$, situated at $\boldsymbol{r}_{\text {acc }}$ with respect to its mass centre, $C_{2}$. Alternatively, material falls onto the primary's surface (dotted line), landing at $B$, located at $\boldsymbol{r}_{\text {acc }}^{*}$ with respect to $\mathcal{C}_{1}$. The unit vectors $\hat{e}_{r}$ and $\hat{e}_{t}$ point along the line joining the two stars (towards the secondary), and perpendicular to this line, respectively, and $v$ is the true anomaly.

the velocity of the accreted material $\boldsymbol{V}_{2}$ with respect to the secondary's mass centre is

$\boldsymbol{V}_{2}=\boldsymbol{W}_{2}-\boldsymbol{v}_{2}$,

where $\boldsymbol{W}_{2}$ is the absolute velocity of the accreted material, and $\boldsymbol{v}_{2}$ is the secondary's orbital velocity. Ejection occurs from the $\mathcal{L}_{1}$ point, located at a distance $r_{\mathcal{L}_{1}}$ from the primary's centre. The Roche radius $R_{\mathcal{L}_{1}}$ and $r_{\mathcal{L}_{1}}$, are calculated as in Davis et al. (2013), using the formalism described by Sepinsky et al. (2007a), that accounts for the donor's rotation.

The impact site is located at $\boldsymbol{r}_{\text {acc }}$ with respect to the secondary's centre of mass. The distance $r_{\text {acc }}$ is either the secondary's radius, $R_{2}$, for direct impact accretion or the accretion disc radius. The latter is estimated from the distance of closest approach of the accretion stream to the secondary, $r_{\min }$, and is given by

$r_{\mathrm{acc}}=1.7 r_{\min }$

(Lubow \& Shu 1975; Ulrich \& Burger 1976), where $r_{\min }$ is determined from our ballistic calculations (see Sect. 2.3). The vector $\boldsymbol{r}_{\text {acc }}$ forms an angle

$\psi=\pi-\phi$,

with the line joining the two stars, $\hat{e}_{r}$ (Fig. 1).

In the theory of osculating elements, the rate of change of the semi-major axis, $\dot{a}$, and of the eccentricity, $\dot{e}$, can be expressed as (see, e.g. Sterne 1960)

$\dot{a}=\frac{P_{\text {orb }}}{\pi\left(1-e^{2}\right)^{\frac{1}{2}}}[\mathcal{S} e \sin v+\mathcal{T}(1+e \cos v)]$ 
and

$\dot{e}=\frac{P_{\mathrm{orb}}\left(1-e^{2}\right)^{\frac{1}{2}}}{2 \pi a}\left\{\mathcal{S} \sin v+\mathcal{T}\left[\frac{2 \cos v+e\left(1+\cos ^{2} v\right)}{1+e \cos v}\right]\right\}$,

where $\mathcal{S}$ and $\mathcal{T}$ are the perturbing forces per unit mass, acting along $\hat{e}_{r}$, and perpendicular to that line (along $\hat{e}_{t}$ ), respectively, and $v$ is the true anomaly.

To account for the fact that the primary's sub-synchronous rotation may cause a fraction $\alpha_{\text {self }}$ of ejected matter to be selfaccreted, we decompose $\mathcal{S}$ and $\mathcal{T}$ into two contributions; that resulting from accretion onto the secondary, and that arising from self-accretion. If $\dot{M}_{1, \text { ej }}^{\text {self }}$ and $\dot{M}_{1, \text { ej }}^{\text {comp }}$ are the mass transfer rates onto the primary and towards the secondary, respectively, then

$\dot{M}_{1, \mathrm{ej}}=\dot{M}_{1, \mathrm{ej}}^{\text {self }}+\dot{M}_{1, \mathrm{ej}}^{\mathrm{comp}}=\alpha_{\text {self }} \dot{M}_{1, \mathrm{ej}}+\left(1-\alpha_{\text {self }}\right) \dot{M}_{1, \mathrm{ej}}$.

The mass accretion rate onto the secondary is $\dot{M}_{2, \text { acc }}=-\beta \dot{M}_{1, \mathrm{ej}}^{\text {comp }}$, where $\beta$ is the accretion efficiency $(\beta=1$ for conservative mass transfer). The corresponding perturbing forces, $\mathcal{S}_{\text {comp }}$ and $\mathcal{T}_{\text {comp }}$, are respectively

$$
\begin{aligned}
\mathcal{S}_{\mathrm{comp}}= & \left(\frac{f_{2, r}}{M_{2}}-\frac{f_{1, r}}{M_{1}}\right)_{\mathrm{comp}}+\frac{\ddot{M}_{2, \mathrm{acc}}}{M_{2}} r_{\mathrm{acc}} \cos \psi-\frac{\ddot{M}_{1, \mathrm{ej}}^{\mathrm{comp}}}{M_{1}} r_{\mathcal{L}_{1}} \\
& +\frac{\dot{M}_{2, \mathrm{acc}}}{M_{2}}\left(V_{2, r}-\omega r_{\mathrm{acc}} \sin \psi\right)-\frac{\dot{M}_{1, \mathrm{ej}}^{\mathrm{comp}}}{M_{1}} V_{1, r},
\end{aligned}
$$

and

$$
\begin{aligned}
\mathcal{T}_{\text {comp }}= & \underbrace{\left.\frac{f_{2, t}}{M_{2}}-\frac{f_{1, t}}{M_{1}}\right)_{\text {comp }}}_{\mathcal{F}_{\text {stream }}^{\text {comp }}}+\underbrace{\frac{\ddot{M}_{2, \text { acc }}}{M_{2}} r_{\text {acc }} \sin \psi}_{\mathcal{H}_{\text {comp }}} \\
& +\underbrace{\frac{\dot{M}_{2, \text { acc }}}{M_{2}}\left(V_{2, t}+\omega r_{\text {acc }} \cos \psi\right)-\frac{\dot{M}_{1, \mathrm{ej}}^{\text {comp }}}{M_{1}}\left(V_{1, t}+\omega r_{\mathcal{L}_{1}}\right)}_{\mathcal{G}_{\text {comp }}},
\end{aligned}
$$

(Hadjidemetriou 1969b; Sepinsky et al. 2007b). The subscripts " $r$ " and " $t$ " refer to the radial and transverse components (i.e. along $\hat{e}_{r}$ and $\hat{e}_{t}$ ) of the vector quantities, respectively. In Eqs. (9) and (10), terms proportional to $\boldsymbol{f}_{i}$ are the gravitational forces per unit mass acting on the $i$ th star due to the mass transfer stream (see Sect. 2.4), and terms proportional to $\dot{M}_{i}$ are associated with the change of linear momentum for the $i$ th star, while terms proportional to $\ddot{M}_{i}$ represent the acceleration of the mass centre of the $i$ th star arising from asymmetric mass loss or gain. In Eq. (10), $\mathcal{F}_{\text {stream }}^{\text {comp }}, \mathcal{G}_{\text {comp }}$ and $\mathcal{H}_{\text {comp }}$ correspond respectively to the gravitational force acting on the secondary by the mass transfer stream, the linear momentum transferred to the secondary and the acceleration of its mass centre, all with respect to the primary (see Sect. 3).

The self-accretion rate back onto the primary is $\dot{M}_{1, \text { acc }}^{\text {self }}=$ $-\dot{M}_{1, \text { ej }}^{\text {self }}$. The associated perturbing forces, $\mathcal{S}_{\text {self }}$ and $\mathcal{T}_{\text {self }}$, are found by considering the total acceleration experienced by the primary resulting from the ejection and re-capture of material (see Appendix A), giving

$$
\begin{aligned}
\mathcal{S}_{\text {self }}= & \left(\frac{f_{2, r}}{M_{2}}-\frac{f_{1, r}}{M_{1}}\right)_{\text {self }}-\frac{\ddot{M}_{1, \mathrm{ej}}^{\text {self }}}{M_{1}}\left(r_{\mathcal{L}_{1}}-r_{\mathrm{acc}}^{*} \cos \psi^{*}\right) \\
& -\frac{\dot{M}_{1, \mathrm{ej}}^{\text {self }}}{M_{1}}\left(V_{1, r}-V_{1, r}^{*}+\omega r_{\mathrm{acc}}^{*} \sin \psi^{*}\right),
\end{aligned}
$$

and

$$
\begin{aligned}
& \mathcal{T}_{\text {self }}=\underbrace{\left(\frac{f_{2, t}}{M_{2}}-\frac{f_{1, t}}{M_{1}}\right)_{\text {self }}}_{\mathcal{F}_{\text {stream }}^{\text {self }}} \underbrace{-\frac{\ddot{M}_{1, \text { ej }}^{\text {self }}}{M_{1}} r_{\text {acc }}^{*} \sin \psi^{*}}_{\mathcal{H}_{\text {self }}} \\
& \underbrace{-\frac{\dot{M}_{1, \text { ej }}^{\text {self }}}{M_{1}}\left(V_{1, t}-V_{1, t}^{*}+\omega r_{\mathcal{L}_{1}}-\omega r_{\mathrm{acc}}^{*} \cos \psi^{*}\right)}_{\mathcal{G}_{\text {self }}},
\end{aligned}
$$

where the asterisks indicate quantities calculated at selfaccretion. Here, $\mathcal{F}_{\text {stream }}^{\text {self }}$ is similar to $\mathcal{F}_{\text {stream }}^{\text {comp }}$ but now for the selfaccreted material, while $\mathcal{G}_{\text {self }}$ and $\mathcal{H}_{\text {self }}$ correspond respectively to the net momentum transferred to the primary by the ejected and self-accreted material, and the acceleration of its centre of mass. The radius $r_{\text {acc }}^{*}$ is determined from the ballistic calculations, and corresponds to the location of the particle where the Roche potential is equal to the potential at the $\mathcal{L}_{1}$-point. Using Eqs. (9) to (12), the total perturbing forces are $\mathcal{S}=\mathcal{S}_{\text {comp }}+\mathcal{S}_{\text {self }}$ and $\mathcal{T}=\mathcal{T}_{\text {comp }}+\mathcal{T}_{\text {self }}$.

For circular orbits, $\dot{a}$ is only a function of $\mathcal{T}$. For infinitesimal changes of $a$ and $e$ over one orbital period, the term in braces in Eq. (7) averages to zero over one orbit, so $\dot{e}=0$ (Hadjidemetriou 1969b). Therefore, in the remainder of Sect. 2, we will just describe the quantities pertinent to the calculation of $\mathcal{T}$.

The total angular momentum of the binary system, $J$, is the sum of the spin angular momenta of each star, $J_{1,2}$, the orbital angular momentum, $J_{\text {orb }}$, and the angular momentum carried by the mass that is not attached to the stars (i.e. the mass in the wind and in the mass transfer stream), $J_{\mathrm{MT}}$, i.e.

$J=J_{1}+J_{2}+J_{\text {orb }}+J_{\mathrm{MT}}=$ const.

The rate of change of the orbital angular momentum, $\dot{J}_{\text {orb }}$, is then determined by taking the time derivative of Eq. (13), and solving for $\dot{J}_{\text {orb }}$, giving

$\dot{J}_{\text {orb }}=-\dot{J}_{1}-\dot{J}_{2}-\dot{J}_{\mathrm{MT}}$,

where

$$
\begin{aligned}
\dot{J}_{\mathrm{MT}}= & \underbrace{-J_{\text {orb }}\left[\frac{\dot{M}_{1, \mathrm{ej}}^{\text {comp }}}{M}\left(\frac{1}{q}-\beta q\right)\right]-m a \mathcal{T}}_{j_{\mathrm{RLOF}}} \\
& \underbrace{-J_{\text {orb }}\left(\frac{\dot{M}_{1, \text { loss }}}{M} \frac{1}{q}+\frac{\dot{M}_{2, \text { loss }}}{M} q\right)}_{j_{\text {lost }}}
\end{aligned}
$$

for circular orbits (see Appendix B). Here, $m=M_{1} M_{2} /\left(M_{1}+\right.$ $M_{2}$ ) is the reduced mass, $\dot{M}_{1,2, \text { loss }}<0$ is the systemic mass loss rate from each star (either via winds and/or non-conservative evolution), $\dot{J}_{\mathrm{RLOF}}$ is the torque resulting from the material transferred between the stars, and $\dot{J}_{\text {lost }}$ is the torque generated by the material leaving the system (and thus associated with $\dot{M}_{1,2, \text { loss }}$ ). The expression for $\dot{J}_{\text {lost }}$ is identical to that of Bonačić Marinović et al. (2008), which considers that the escaping material carries the specific orbital angular momentum of the star.

For brevity, we term our formalism the osculating scheme. If the stars are point masses, and the gravitational attraction exerted by the accretion stream is neglected, as is usually assumed, then for conservative mass transfer $\dot{J}_{\mathrm{MT}}=0$ in Eq. (15) and we recover the classical formulation ${ }^{1}$.

1 For conservative mass transfer and neglecting the stellar spins, inserting Eqs. (15) and (7) into Eq. (1) recovers Eq. (6). 


\subsection{Stellar torques}

The torques acting on the $i$ th star can be decomposed into the tidal torque $\dot{J}_{\text {tide }, i}$, and the torque arising from mass ejection or accretion, $\dot{J}_{\dot{M}_{i}}$, to give

$\dot{J}_{i}=\dot{J}_{\text {tide }, i}+\dot{J}_{\dot{M}_{i}}$.

For stars with radiative envelopes, we apply the prescription for dynamical tides described by Zahn (1989). For convective stars, we use the formalism of Zahn (1977) describing equilibrium tides (see Siess et al. 2013, for further details). For $\dot{J}_{\dot{M}_{i}}$ we have

$\dot{J}_{\dot{M}_{i}}=\dot{M}_{i}\left(\omega r_{i}^{2}+U_{i, t} r_{i} \cos \phi-U_{i, r} r_{i} \sin \phi\right)$,

(Piotrowski 1964; Flannery 1975) where $U_{i, t}$ and $U_{i, r}$ are the tangential and radial components of the ejection or accretion velocity with respect to a frame of reference co-rotating with the binary, $r_{i}$ is the distance from the $i$ th star's mass centre to the mass ejection/accretion point (i.e. $r_{\mathcal{L}_{1}}$ or $r_{\text {acc }}$ ), $\phi$ is the angle between the ejection/accretion point and the line joining the two stars, and $\dot{M}_{i}$ is the mass loss/accretion rate. From Eq. (17), the torque applied onto the primary because of mass ejection is

$\dot{J}_{\dot{M}_{1}}=-\left|\dot{M}_{1, \mathrm{ej}}\right|\left(\omega r_{\mathcal{L}_{1}}^{2}+U_{1, t} r_{\mathcal{L}_{1}}\right)$,

where we have used the fact that $\phi=0$. The torque arising from self-accretion is

$\dot{J}_{\dot{M}_{1}}^{\text {self }}=\dot{M}_{1, \text { acc }}^{\text {self }}\left[\omega\left(r_{\mathrm{acc}}^{*}\right)^{2}+U_{1, t}^{*} r_{\mathrm{acc}}^{*} \cos \phi^{*}-U_{1, r}^{*} r_{\mathrm{acc}}^{*} \sin \phi^{*}\right]$,

while the torque applied onto the secondary is

$\dot{J}_{\dot{M}_{2}}=\dot{M}_{2, \mathrm{acc}}\left(\omega r_{\mathrm{acc}}^{2}+U_{2, t} r_{\mathrm{acc}} \cos \phi-U_{2, r} r_{\mathrm{acc}} \sin \phi\right)$.

As shown by Packet (1981), accretion may rapidly spin up the secondary to its critical angular velocity $\Omega_{2}^{\text {crit }}=\left(G M_{2} / R_{2}^{3}\right)^{1 / 2}$. Deschamps et al. (2013) argued that super-critical rotation can be avoided either by the interaction between the secondary and an accretion disc, or magnetic braking (wind braking and disclocking). For simplicity, we mimic these mechanisms by forcing the secondary's spin to remain below $\Omega_{2}^{\text {crit }}$. This translates into an effective torque on the gainer given by

$\dot{J}_{\dot{M}_{2}}^{\text {eff }}=\min \left(\frac{I_{2} \Omega_{2}^{\text {crit }}-J_{2,0}}{\Delta t}, \dot{J}_{\dot{M}_{2}}\right)$

where $\Delta t$ is the evolutionary time step, which is constrained using the nuclear burning timescales, changes in the stars' structures, the rates of change of the orbital parameters and the mass transfer rate (see Siess et al. 2013, for further details). Also, $I_{2}$ is the secondary's moment of inertia and $J_{2,0}$ is the secondary's angular momentum at the previous time step. For simplicity, we assume that each star rotates as a solid body, since the treatment of differential rotation is beyond the scope of this investigation (but see Sect. 4).

\subsection{Ejection and accretion velocities}

The components of $\boldsymbol{V}_{1}$ and $\boldsymbol{V}_{2}$ along $\hat{e}_{t}$, i.e. $V_{1, t}$ and $V_{2, t}$, respectively are given by (Hadjidemetriou 1969b)

$V_{1, t}=U_{1, t}+\omega r_{\mathcal{L}_{1}}$,

and

$V_{2, t}=U_{2, t}+\omega r_{\mathrm{acc}} \cos \psi$.
For particles ejected from the $\mathcal{L}_{1}$ point, we set $U_{1, r}$ to the sound speed, $c_{\mathrm{s}}$, at the primary's photosphere, and $U_{1, t}$ is calculated from

$U_{1, t}=r_{\mathcal{L}_{1}}\left(\Omega_{1}-\omega\right)$,

where $\Omega_{1}$ is the spin angular velocity of the primary. We evaluate $\psi, U_{2, r}$ and $U_{2, t}$ by solving the restricted three-body equations of motion (e.g. Hadjidemetriou 1969a; Flannery 1975).

\subsection{Perturbing forces from the accretion stream}

We discretize the mass transfer stream as a succession of individual particles of mass $\delta m_{i}$. The force exerted onto the $j$ th star by the $i$ th particle is

$\boldsymbol{f}_{j i}=\frac{G M_{j} \delta m_{i}}{r_{j i}^{3}} \boldsymbol{r}_{j i}$

where $r_{j i}$ is the distance between the $i$ th particle and star $j$. In the transverse direction, we have for the primary

$r_{1 i}=\left\{\left[x_{i}-(1-\mu) a\right]^{2}+y_{i}^{2}\right\}^{\frac{1}{2}}$

and for the secondary

$r_{2 i}=\left[\left(x_{i}-\mu a\right)^{2}+y_{i}^{2}\right]^{\frac{1}{2}}$

where $\mu \equiv M_{1} /\left(M_{1}+M_{2}\right)$ and $\left(x_{i}, y_{i}\right)$ are the coordinates of particle $i$ in the co-rotating frame. Summing Eq. (25) over all stream particles, and taking the difference between the gravitational force (per unit mass) acting on the secondary and on the primary star gives

$\mathcal{F}_{\text {stream }}=\frac{f_{2, t}}{M_{2}}-\frac{f_{1, t}}{M_{1}}=G \sum_{i} \delta m_{i} y_{i}\left(\frac{1}{r_{2 i}^{3}}-\frac{1}{r_{1 i}^{3}}\right)$.

During a time-step $\delta t$ of the ballistic calculation, the mass contained in the stream that falls towards the secondary is given by

$\delta m_{i}^{\mathrm{comp}}=-\dot{M}_{1, \mathrm{ej}}^{\mathrm{comp}} \delta t$.

Inserting Eq. (29) into Eq. (28), then in the limit $\delta t \rightarrow 0, \mathcal{F}_{\text {stream }}^{\text {comp }}$ writes as

$\mathcal{F}_{\text {stream }}^{\text {comp }}=-G \dot{M}_{1, \text { ej }}^{\text {comp }} \int_{0}^{\tilde{t}_{\text {comp }}} y_{\text {comp }}\left(\frac{1}{r_{2}^{3}}-\frac{1}{r_{1}^{3}}\right)_{\text {comp }} \mathrm{d} t$.

Similarly for the stream falling back onto the primary, we have

$\delta m_{i}^{\text {self }}=-\dot{M}_{1, \mathrm{ej}}^{\text {self }} \delta t$

giving

$\mathcal{F}_{\text {stream }}^{\text {self }}=-G \dot{M}_{1, \mathrm{ej}}^{\text {self }} \int_{0}^{\tilde{t}_{\text {self }}} y_{\text {self }}\left(\frac{1}{r_{2}^{3}}-\frac{1}{r_{1}^{3}}\right)_{\text {self }} \mathrm{d} t$.

In Eqs. (30) and (32), $\tilde{t}$ is the particle's travel time between the $\mathcal{L}_{1}$ point and the point of impact, and $r_{1}, r_{2}$, and $y$ describe the position of the particle at time $t$, given by our integration of the ballistic trajectories. The subscripts "comp" and "self" indicate quantities pertaining to material falling onto the secondary and onto the primary, respectively. 


\subsection{Treatment of the mass transfer stream}

The finite width of the matter stream that is ejected from the $\mathcal{L}_{1}$-point is accounted for when calculating the quantities found in Eqs. (10) and (12) as described below. For a primary with an effective temperature $T_{\text {eff, } 1}$, mean molecular weight at the photosphere $\mu_{\mathrm{ph}, 1}$, the surface area, $\mathcal{A}$, of the stream at the $\mathcal{L}_{1}$ point is

$\mathcal{A}=\frac{2 \pi \mathcal{R} T_{\mathrm{eff}, 1}}{\mu_{\mathrm{ph}, 1}} \frac{a^{3} q}{G M_{1}}\{g(q)[g(q)-f-q f]\}^{-1 / 2}$,

(Davis et al. 2013) where $\mathcal{R}$ is the ideal gas constant. In Eq. (33),

$$
g(q)=\frac{q}{\left(r_{\mathcal{L}_{1}} / a\right)^{3}}+\frac{1}{\left[1-\left(r_{\mathcal{L}_{1}} / a\right)\right]^{3}},
$$

(Kolb \& Ritter 1990) and

$$
f=\frac{\Omega_{1}}{\omega}
$$

corrects for any effects arising from the primary's rotation.

We then calculate the ballistic trajectory of $N$ particles, which are ejected at evenly spaced intervals, $\Delta \ell$, along the width of the $\mathcal{L}_{1}$-point, $\ell \approx \sqrt{\mathcal{A}}$. To account for the Gaussian density distribution of the particles at the $\mathcal{L}_{1}$ nozzle (see, e.g. Lubow \& Shu 1975; Edwards \& Pringle 1987; Raymer 2012) we weight each trajectory using

$\xi(\tilde{\ell})=\eta \mathrm{e}^{-\frac{\tilde{\ell}^{2}}{2 \sigma^{2}}}$,

where $-1 \leq \tilde{\ell} \leq 1$ is the normalised distance along the finite width of the stream (the $\mathcal{L}_{1}$-point is located at $\tilde{\ell}=0$ ), $\sigma$ is the standard deviation and $\eta$ is a normalisation constant set by the requirement that $\int_{-1}^{1} \xi(\tilde{\ell}) \mathrm{d} \tilde{\ell}=1$. We use $\sigma=0.4$ so that the density at $\tilde{\ell}=-1$ and $\tilde{\ell}=1$ is equal to the donor's photospheric density, and use $N=128$ particles. We checked that increasing $N$ any further or slightly varying $\sigma$ has negligible impact on the calculations.

Since we are dealing with more than one particle landing on each star, we calculate the average stream properties as follows. Let $Q_{k}^{\text {comp }} \in\left\{\mathcal{F}_{\text {stream }, k}^{\text {comp }}, U_{1,2, t, k}, r_{\text {acc }, k}, \psi_{k}\right\}$ be some quantity related to the $k$ th particle that is ejected from $\tilde{\ell}_{k}$, which is subsequently accreted by the companion. Then the mean value of this quantity for a given model is calculated using

$$
\left\langle Q^{\mathrm{comp}}\right\rangle=\frac{\sum_{k=1}^{N_{\text {comp }}} Q_{k}^{\mathrm{comp}} \xi\left(\tilde{\ell_{k}}\right) \Delta \tilde{\ell}}{\sum_{k=1}^{N_{\text {comp }}} \xi\left(\tilde{\ell_{k}}\right) \Delta \tilde{\ell}}
$$

where $N_{\text {comp }}$ is the number of particles landing on the companion. A similar expression for the particles landing on the donor is used, where we replace "comp" with "self". Hence, we replace $\mathcal{F}_{\text {stream }}^{\text {self,comp }}, U_{1,2, t}, U_{1, t}^{*}, r_{\text {acc }}^{(*)}$ and $\psi^{(*)}$ in Eqs. (10) and (12) with the corresponding mean values, as determined by Eq. (37). Finally, the fraction of particles landing back on the donor star is

$\alpha_{\text {self }}=\sum_{k=1}^{N_{\text {self }}} \xi\left(\tilde{\ell}_{k}\right) \Delta \tilde{\ell}$

where $N_{\text {self }}$ is the number of particles undergoing self-accretion $\left(N=N_{\text {self }}+N_{\text {comp }}\right)$.

\subsection{The binary models}

To analyse the impact of this new formalism on the evolution of Algols, we consider two systems. The first configuration is a $5+3 M_{\odot}$ binary, with an initial period $P_{i}=7$ days, undergoing case $\mathrm{B}$ mass transfer (during shell H-burning). Our second system is a $6+4 M_{\odot}$ binary, $P_{i}=2.5$ days, which commences mass exchange during core $\mathrm{H}$-burning (case A). We assume a solar composition $(Z=0.02)$, and apply moderate convective core overshooting, with $\alpha_{\mathrm{ov}}=\Lambda / H_{\mathrm{p}}=0.2$, where $\Lambda$ is the mixing length and $H_{\mathrm{p}}$ is the pressure scale height. The initial spin periods of each star are set to the initial orbital periods at the start of the simulation (i.e. $f=1$ in Eq. (35)) and the orbits are circular.

\section{Results}

Section 3.1 presents our osculating calculations for the case B system, which are compared to calculations determined from the classical scheme (Sect. 3.1.1), followed by case BB (shell He-burning) mass transfer in Sect. 3.1.2. Calculations for the case A model are given in Sect. 3.2.

\section{1. $5+3 M_{\odot}, P_{\mathrm{i}}=7$ days}

\subsubsection{Case B mass transfer}

As the primary evolves off the main sequence, its radius and moment of inertia increase on a much shorter timescale $\left(\tau_{R}=\right.$ $\left.R / \dot{R} \approx 10^{8} \mathrm{yr}\right)$ than tides can maintain synchronization $\left(\tau_{\text {sync }}=\right.$ $\left|\Omega_{1}-\omega\right| / \dot{\omega} \approx 10^{10} \mathrm{yr}$ ) and $\Omega_{1}$ declines (Fig. 2a). Therefore, mass transfer starts while the primary star is rotating significantly subsynchronously, and this has two important consequences. First, the Roche radius calculated using the Sepinsky et al. (2007a) formalism is 7 per cent larger than the Eggleton (1983) prescription. Consequently, the primary must evolve further along the subgiant branch before it can start transferring mass. Secondly, at the onset of RLOF, all the material ejected by the primary star is initially self-accreted ( $\alpha_{\text {self }}=1$, panel b, solid black curve). This process induces a positive torque onto the primary (Eq. (19)), increasing $\Omega_{1}$ (dotted green curve) and thus $U_{1, t}$, causing material to progressively flow onto the companion $\left(\alpha_{\text {self }}<1\right)$. In parallel, mass ejected through the $\mathcal{L}_{1}$-point applies a negative torque onto the primary (Eq. (18)). Since, initially, more material is falling onto the primary than onto the companion, the net effect is an acceleration of the primary's rotation rate, i.e. $\left|\dot{J}_{\dot{M}_{1}}\right|<\dot{J}_{\dot{M}_{1}}^{\text {sslf }}$, and a reduction in the amount of self-accreted material. As $\Omega_{1}$ increases, $\dot{J}_{\dot{M}_{1}}^{\text {self }}$ drops until eventually the net torque applied onto the primary via the ejection and self-accretion process is zero, i.e. $\left|\dot{J}_{\dot{M}_{1}}\right| \approx j_{\dot{M}_{1}}^{\text {self }}$. This situation is characterized by a plateau in $\alpha_{\text {self }} \approx 0.2$. Throughout the self-accretion process, the mass loss timescale $\tau_{\dot{M}}=M_{1} /\left|\dot{M}_{1}\right|<\tau_{\text {sync }}$, so tides are unable to enforce synchronous rotation (dashed, blue curve).

For circular orbits, Eq. (6) reduces to

$\dot{a}=\frac{P_{\text {orb }}}{\pi} \mathcal{T}=\frac{P_{\text {orb }}}{\pi}\left(\mathcal{T}_{\text {comp }}+\mathcal{T}_{\text {self }}\right)$,

and so the sign of $\dot{a}$ depends on the signs of $\mathcal{T}_{\text {comp }}$ and $\mathcal{T}_{\text {self }}$. To understand the evolution of the separation, we show in Fig. 3 the various contributions entering the expression for $\mathcal{T}$, i.e. the gravitational force applied onto the secondary (primary) by the ejected particle $\mathcal{F}_{\text {stream }}^{\text {comp,(self) }}$, and the linear momentum transferred

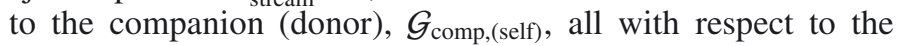
donor. Throughout mass transfer, the terms $\mathcal{H}_{\text {self }}$ and $\mathcal{H}_{\text {comp }}$, 




Fig. 2. Evolution in time (since the start of mass transfer at $t_{\mathrm{RLOF}}=9.62034 \times 10^{7} \mathrm{yr}$ ) of the primary's spin angular velocity in units of the orbital velocity, $\Omega_{1} / \omega$ (dotted green curve, left axis), for the $5+3 M_{\odot}$ system, initial period $P_{i}=7 \mathrm{~d}$, using the osculating formalism. Panel a): as the primary evolves off the main sequence up to the onset of mass transfer; panel $\mathbf{b}$ ): during the self-accretion phase; panel c): during the slow mass transfer phase. Panel b) also shows the fraction of ejected material that falls back onto the primary, $\alpha_{\text {self }}$ (solid black curve, left axis), and the ratio of the tidal synchronization timescale to the mass transfer timescale, $\tau_{\text {sync }} / \tau_{\dot{M}}$ (dashed blue curve, right axis). Note the change in scales along the $\mathrm{x}$-axis in each panel. The top panels indicate the evolution of $q=M_{1} / M_{2}$, and the open red square indicates where $q=1$.
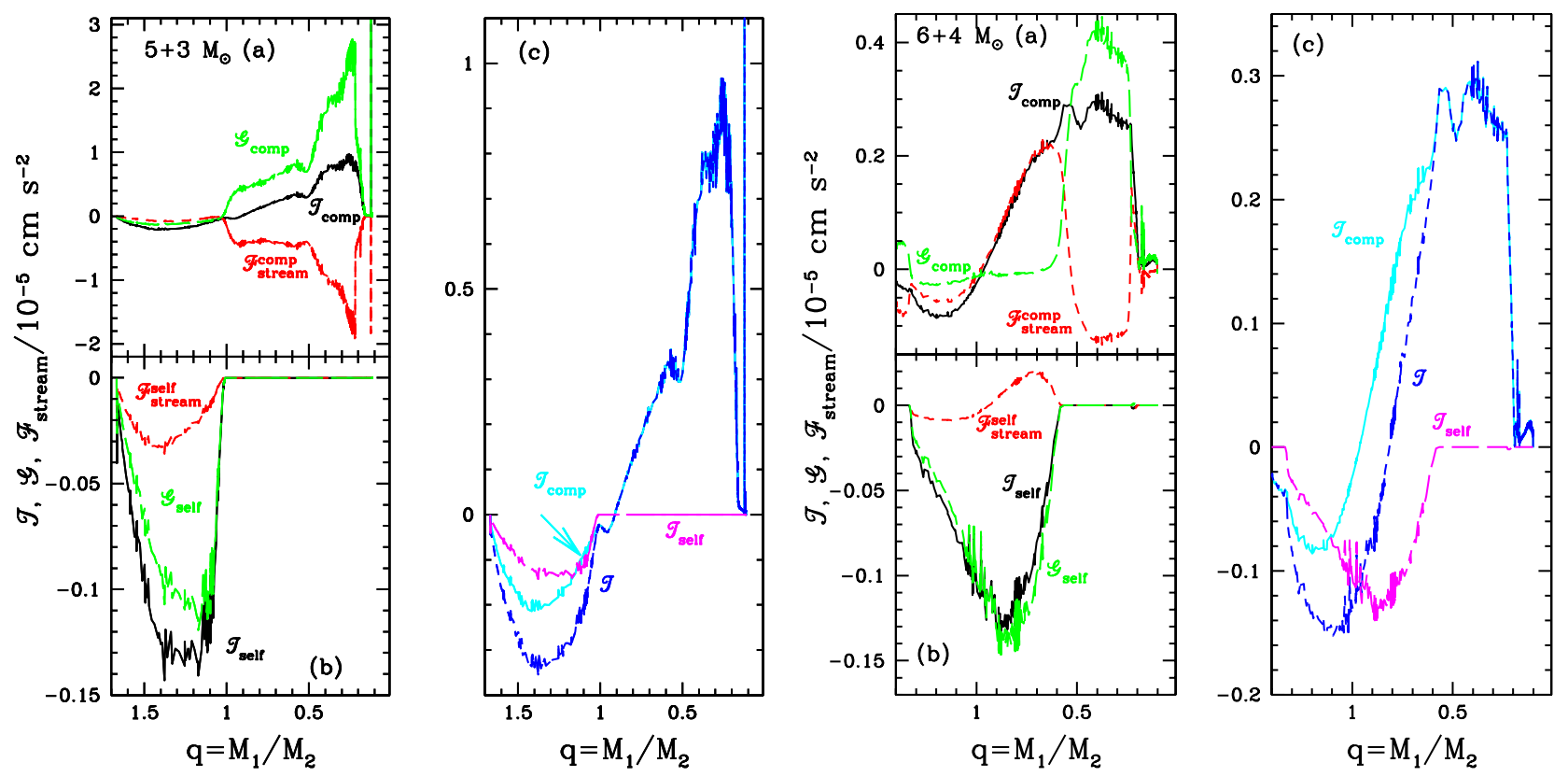

Fig. 3. Left: evolution for the $5+3 M_{\odot}$ binary system with $P_{i}=7 \mathrm{~d}$ of $\mathcal{T}_{\text {comp,self }}$ (solid black curve), $\mathcal{G}_{\text {comp,self }}$ (long-dashed green) and $\mathcal{F}_{\text {stream }}^{\text {complf }}$ (short-dashed red) arising from mass transfer onto the companion (panel a)) and self-accretion (panel b)). Panel c) compares $\mathcal{T}_{\text {self }}($ long-dashed magenta), $\mathcal{T}_{\text {comp }}\left(\right.$ solid cyan) and the total $\mathcal{T}$ (short-dashed blue). Right: the same but for the $6+4 M_{\odot}$ system, $P_{i}=2.5 \mathrm{~d}$. 
corresponding to the acceleration of the donor's or companion's mass centre, are negligible because $\ddot{M}_{1, \mathrm{ej}} \approx 0$, and for this reason it is not displayed in Fig. 3. At the start of mass transfer, the

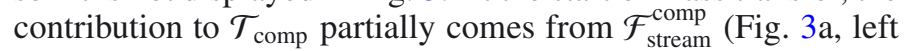
panels, red short-dashed curves), which is negative for two reasons. Firstly, the particles are located at $y_{\text {comp }}<0$ and secondly they are typically situated in the vicinity of the secondary i.e. $1 / r_{2}>1 / r_{1}$ (see Eq. (30)).

The $\mathcal{G}_{\text {comp }}$ term (green, long-dashed curves) is small for $q>1$, but increases as $q$ declines. Indeed, as mass transfer proceeds, the primary's Roche lobe radius and therefore $r_{\mathcal{L}_{1}}$ shrink, and so the $\mathcal{L}_{1}$ point moves further away from the companion's surface. A particle's travel time thus rises and it can accelerate to a larger impact velocity $\left(V_{2, t}\right)$. Even though $\mathcal{G}_{\text {comp }}$ also depends on $V_{1, t}+\omega r_{\mathcal{L}_{1}}$, its magnitude is about a factor of 10 smaller than the $\left|V_{2, t}+\omega r_{\text {acc }} \cos \psi\right|$ term.

During self-accretion, $\mathcal{T}_{\text {self }}$ is dictated by $\mathcal{G}_{\text {self }}$ (Fig. 3b). We find that $V_{1, t} \approx-1 \times 10^{7} \mathrm{~cm} \mathrm{~s}^{-1}$ and $V_{1, t}^{*} \approx 8 \times 10^{6} \mathrm{~cm} \mathrm{~s}^{-1}$, which, for small $\psi^{*}$, gives $\mathcal{T}_{\text {self }}<0$ (Eq. (12)). Similarly to $\mathcal{F}_{\text {stream }}^{\text {comp }}$, $\mathcal{F}_{\text {stream }}^{\text {self }}<0$ because $y_{\text {self }}<0$ and, owing to the large value of $r_{\mathcal{L}_{1}}$ for $q>1$, the particle is closer to the secondary than to the primary.

Hence, self-accretion enhances the rate of shrinkage of both the orbital separation (Fig. 3c) and the primary's Roche radius. This faster contraction of $R_{\mathcal{L}_{1}}$ increases the overfilling factor resulting in a mass transfer rate that is a factor of $\sim 1.6$ higher than in the classical scheme. In response to this higher mass loss rate, the primary's radiative layers further contract and it attains a lower surface luminosity on the Hertzsprung-Russel (HR) diagram compared to the classical calculation (Fig. 4a).

To quantify the impact of self-accretion, we re-ran a simulation by setting $\mathcal{T}_{\text {self }}=0$, as indicated by the short-dashed green curve in Fig. 4d. Differences in the mass transfer rate and the evolution along the HR diagram are negligible. However, the post-mass transfer orbital period (about $21 \mathrm{~d}$ ) is slightly longer than when self-accretion is included (17 d); a relative difference of about 20 per cent.

As mass transfer proceeds, $\tau_{\text {sync }}$ decreases as a result of the deepening of the primary's surface convection zone in response to mass loss (Webbink 1977a). Eventually, $\tau_{\text {sync }}<\tau_{\dot{M}}$ (Fig. 2b), and tides can re-synchronize the primary. Moreover, as the primary's mass declines, it exerts a smaller gravitational attraction onto the ejected particle, and evermore material falls onto the secondary star, as indicated by the decrease in $\alpha_{\text {self }}$.

Once $q \lesssim 1$, self-accretion shuts off and soon after (when $q \simeq 0.9) \mathcal{T}_{\text {comp }}$ becomes positive, allowing the orbit to expand. The rise in $\mathcal{T}_{\text {comp }}$ is because of the aforementioned growth of the $\mathcal{G}_{\text {comp }}$ term, resulting from the higher impact velocity $\left(V_{2, t}\right)$. Eventually, the primary restores thermal equilibrium, $\dot{M}_{1}$ declines (Fig. 4c) and the evolution enters the slow phase (around $q \approx 0.16$ ), where mass transfer occurs on the nuclear timescale of the hydrogen-burning shell (Paczyński 1971). The calculated mass transfer rates for the classical and osculating models are virtually identical, since the shell-burning properties in both cases are the same ${ }^{2}$. Also note that throughout the slow phase, $\tau_{\text {sync }} \ll \tau_{\dot{M}}$ and so tides can enforce synchronous rotation of the primary (Fig. 2c).

By the end of the simulations, the difference in the periods is significant with 17 days for the osculating scheme compared to 71 days for the classical model. The primary's radius is correspondingly smaller, since it keeps track of its Roche lobe, which

\footnotetext{
2 The spike just before mass transfer terminates is due to the ignition of core Helium burning.
}

is a function of $a$. This explains why, for a given luminosity, the osculating model gives a hotter primary than in the classical case (Fig. 4a).

The shorter orbital period predicted by the osculating scheme is caused by the negative $\mathcal{F}_{\text {stream }}^{\text {comp }}$ contribution. Indeed, neglecting this term gives $\mathcal{T}_{\text {comp }}=\mathcal{G}_{\text {comp }}$, yielding the longest postmass transfer orbital periods out of all our calculations (Fig. 4d, long-dashed blue curve). In this case, the Roche filling primary has a larger radius and a lower effective temperature, causing a substantial surface convection zone to develop (radial extent of about $60 R_{\odot}$ ). This enhances the mass transfer rate because convective layers expand upon mass loss, causing the star to over-fill its Roche lobe further.

When He ignites in the primary, mass transfer terminates and the final masses are virtually identical between the classical and osculating schemes $\left(M_{1} \approx 0.87 M_{\odot}, M_{2} \approx 7.3 M_{\odot}\right)$.

Both the osculating and classical formalisms predict similar evolutionary tracks of the secondary on the HR diagram (Fig. 4b). Once mass transfer enters the slow phase, the secondary relaxes towards thermal equilibrium, establishing a new effective temperature and luminosity along the main sequence which is appropriate for its new mass.

\subsubsection{Case BB mass transfer}

Once mass transfer has stopped, the structure of the primary consists of a convective He-burning core of $0.27 M_{\odot}$, surrounded by a radiative envelope of $\sim 0.60 M_{\odot}$. The H-burning shell is located at mass coordinate $M_{r} \approx 0.73 M_{\odot}$, and has a mass of about $0.06 M_{\odot}$. When He ignites in the core, the primary contracts within its Roche lobe on a timescale much shorter than $\tau_{\text {sync }}$ leading to super-synchronous rotation (Fig. 5, left panel, solid green curve). The star accelerates up to approximately 3 per cent of the critical velocity when $\Omega_{1} / \omega \approx 12$. These calculations therefore predict the presence of rapidly rotating core-He burning stars in detached binaries. To the best of our knowledge, there are no available observations of such systems in this evolutionary phase.

At about $2.72 \times 10^{7} \mathrm{yr}$ since the start of case B mass transfer, the activation of shell-He burning produces a rapid expansion of the primary. As the star fills more of its Roche lobe $\left(R_{1} / R_{\mathcal{L}, 1}\right.$, dashed cyan curve) the tidal forces strengthen and within $\sim 1.5 \times 10^{6} \mathrm{yr}$, the primary is re-synchronized. The ensuing case BB (Fig. 5, shaded region) is characterized by a constant mass exchange rate of about $10^{-7} M_{\odot} \mathrm{yr}^{-1}$, and an expansion of the orbit because $q<1$. Synchronous rotation is maintained during the entire phase of mass transfer, so no self-accretion occurs. In both schemes, mass transfer lasts for about $3 \times 10^{5} \mathrm{yr}$.

Mass transfer ceases as a result of re-ignition of the H-burning shell, which causes the primary's radius to shrink. At this point, the binary consists of a $0.8 M_{\odot} \mathrm{CO}$ star, undergoing $\mathrm{H}$-shell burning in the surface layers, and a $7.2 M_{\odot}$ main sequence companion. The final orbital periods are approximately 80 days and 19 days for the classical and osculating calculations, respectively.

To calculate the subsequent evolution of this system is beyond the scope of the investigation. Nonetheless, we can infer its fate based on the binary parameters. Eventually, the secondary star will evolve off the main sequence, fill its own Roche lobe and transfer mass back to the CO primary star. For the osculating scheme, we estimate that the secondary will fill its Roche lobe with a radius of about $34 R_{\odot}$ as it is crossing the sub-giant branch, and for the classical scheme, when the star approaches the base of the giant branch with a radius of $\sim 60 R_{\odot}$. Because 

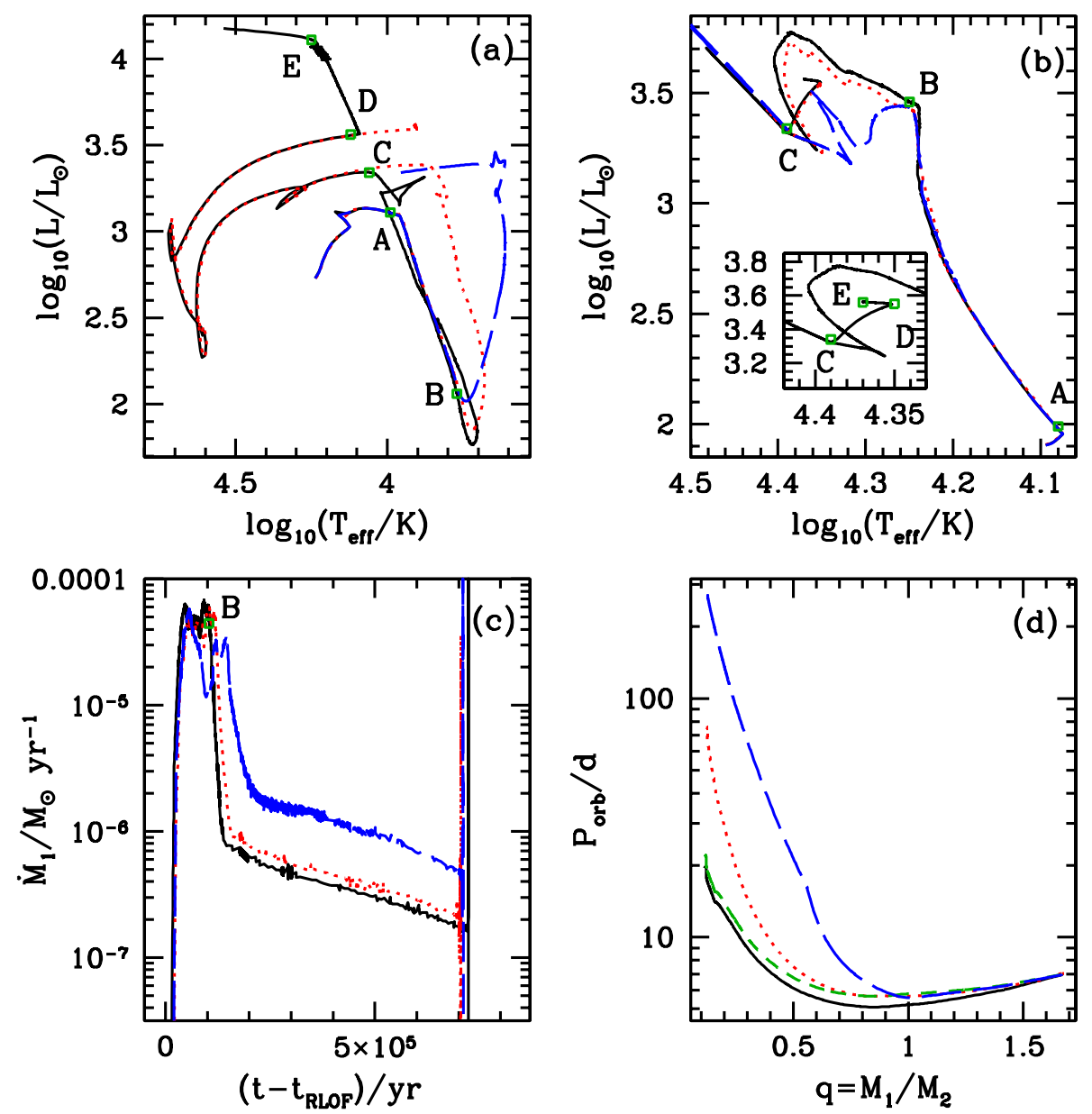

Fig. 4. Evolution of the $5+3 M_{\odot}$ system, $P_{i}=7 \mathrm{~d}$, calculated using the osculating (solid black curves) and the classical (dotted red curves) prescriptions. The short-dashed green and the long-dashed blue curves also use the osculating scheme, but $\mathcal{T}_{\text {self }}$ and $\mathcal{F}_{\text {stream }}$ have been set to zero, respectively. For clarity, the dashed green curve has been omitted from panels a) to c) as it is indistinguishable from the black curve. The longdashed blue curve has been truncated in panel a), since it follows the same track as the black and red curves during core-He burning. Panels a) and b) show the evolutionary path in the Hertzsprung-Russel diagram of the primary and secondary. Panel c) shows the mass loss rate, $\dot{M}_{1}$, as a function of time since mass transfer started and panel d) the evolution of the orbital period, $P_{\text {orb }}$, as a function of the mass ratio, $q=M_{1} / M_{2}$. The different evolutionary phases are indicated by the open green squares, and are labelled as follows: A: start of case B mass transfer; B: $q=1$; C: end of case B mass transfer (coincides with the end of H-shell burning); D: start of case BB mass transfer (onset of He-core burning); E: end of case BB mass transfer (onset of He-shell burning).

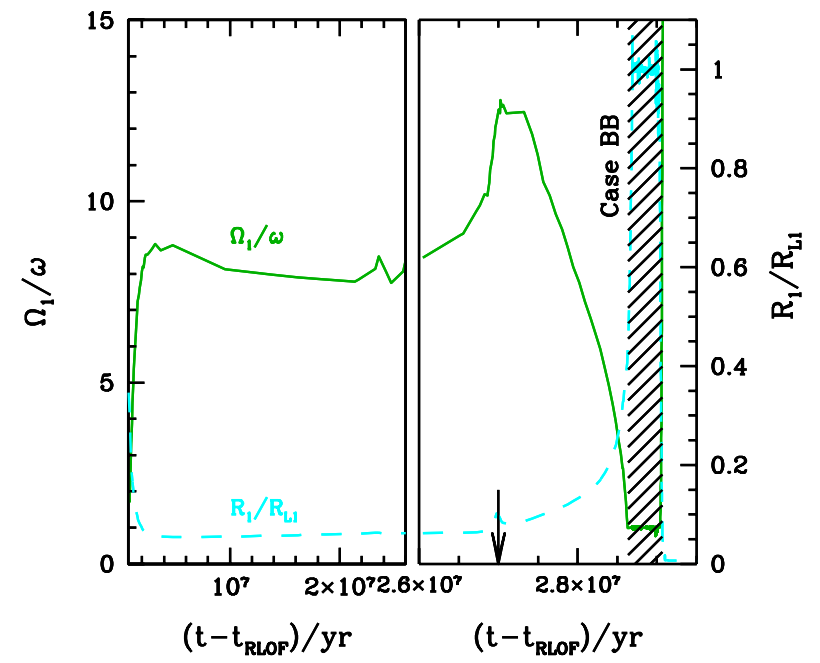

Fig. 5. Similar to Fig. 2, but now during core He burning (left panel), and shell He-burning (right panel), the onset of which is indicated by the arrow. The dashed cyan curve shows $R_{1} / R_{\mathcal{L}, 1}$ (right axis), and the shaded region marks case BB mass transfer. of the high mass ratio $M_{2} / M_{1} \approx 8$, which lies well above the critical value of between 1.2 and 1.3 for dynamically unstable mass transfer (Webbink 2008), we therefore expect this system to enter common envelope evolution.

\subsubsection{Effect of changing the initial orbital period}

Figure 6a shows that $\alpha_{\text {self }}$ levels off to between 0.15 and 0.2 for the considered values of $P_{i}$. In addition, the duration of the self-accretion phase progressively decreases, from $5.2 \times 10^{4}$ to $2.6 \times 10^{4} \mathrm{yr}$ when the initial period, $P_{i}$, rises from 7 to $13 \mathrm{~d}$. By increasing $P_{i}$, the primary evolves further along the sub-giant branch before mass transfer starts, and can develop a deeper convection zone. When RLOF occurs, tides are more efficient at resynchronizing the primary, and self-accretion is stopped earlier.

For $P_{i}$ between 7 and 13 days, $\alpha_{\text {self }} \approx 1$ at the start of mass transfer. By contrast, for $P_{i}=3 \mathrm{~d}, \alpha_{\text {self }}$ rises from zero to a constant value of approximately 0.2 . In this case, the primary is still very close to its main sequence location in the HR diagram, and it is initially not rotating sufficiently slowly to trigger 
P. J. Davis et al.: Binary evolution using the theory of osculating orbits. I.
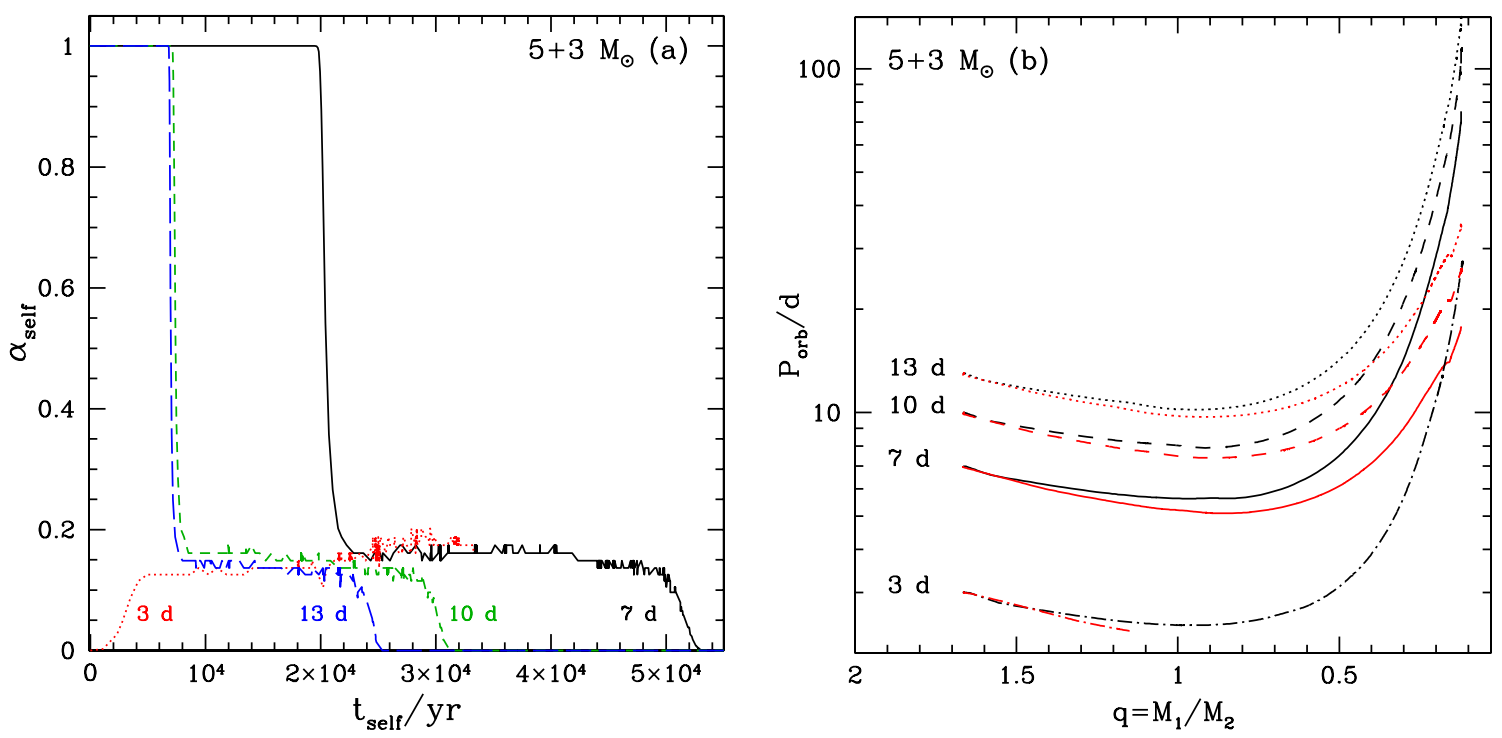

Fig. 6. a) Evolution of $\alpha_{\text {self }}$ as a function of time, $t_{\text {self }}$, since the start of self-accretion for a $5+3 M_{\odot}$ system, with an initial orbital period, $P_{i}$ of $3 \mathrm{~d}$ (dotted red curve), $7 \mathrm{~d}$ (solid black), $10 \mathrm{~d}$ (green short dashed) and $13 \mathrm{~d}$ (blue long-dashed). b) Evolution of the orbital period, $P_{\text {orb }}$, as a function of $q=M_{1} / M_{2}$, for different initial orbital periods: $3 \mathrm{~d}$ (dot-dashed); $7 \mathrm{~d}$ (solid); $10 \mathrm{~d}$ (short-dashed) and $13 \mathrm{~d}$ (dotted). Black curves refer to classical calculations, and the red lines to the osculating scheme.

self-accretion. Only once enough angular momentum has been removed from the primary does self-accretion occur.

The final primary masses are between 0.85 and $0.88 M_{\odot}$ and the secondary masses between 7.15 and $7.12 M_{\odot}$, irrespective of whether the classical or the osculating scheme is used. However, the osculating scheme systematically yields shorter post-mass transfer orbital periods, by a factor of about 4 (Fig. 6b), and for the model with $P_{i}=3 \mathrm{~d}$, it gives rise to a contact system, in contrast to the classical scheme.

\section{2. $6+4 M_{\odot}, P_{\mathrm{i}}=2.5 d$}

Since the initial period is shorter for this model, tides are able to establish synchronous rotation by the time the primary fills its Roche lobe (Fig. 7a). Therefore, all ejected mass is initially accreted onto the companion $\left(\alpha_{\text {self }}=0\right)$ and only once mass ejection has removed a sufficient amount of angular momentum from the primary does self-accretion occur, with $\alpha_{\text {self }} \approx 0.15$.

As we remarked, self-accretion enhances the orbital contraction because of the negative contribution from $\mathcal{G}_{\text {self }}$ (right panel, Fig. 3b). In contrast to the case B model, this process continues after the mass ratio has reversed and only ceases when $q \approx 0.6$. The reason for this difference stems from the time delay associated with the appearance of a surface convection zone, which reinforces the tidal interaction and accelerates the primary's rotation velocity back to synchronous rotation. This persisting selfaccretion episode maintains $\mathcal{T}_{\text {self }}<0$ for a longer period of time and the orbit contracts until $q \approx 0.8$ (Fig. 3c). Also note that $\mathcal{F}_{\text {stream }}^{\text {self }}>0$ when $q<1$ because the ejected material is typically located in the vicinity of the primary $\left(1 / r_{1}>1 / r_{2}\right.$ in Eq. (32)), owing to the close proximity of the $\mathcal{L}_{1}$-point to the primary. The positive value for $\mathcal{F}_{\text {stream }}^{\text {comp }}$, on the other hand, is because the primary's sub-synchronous rotation deflects the particles such that they have $y_{\text {comp }}>0$.

At $t \approx t_{\mathrm{RLOF}}+1 \times 10^{6} \mathrm{yr}(q \approx 0.3)$, the convection zone recedes and $\tau_{\text {sync }}$ rises again (Fig. $7 b$ ). Subsequent mass ejection brings the primary back into sub-synchronous rotation, triggering a second self-accretion episode, with about 10 per cent of the ejected material falling back onto the star. This second self-accretion event is absent in the case B model because of the higher sound speed $\left(U_{1, r}\right)$ for the primary. The impact of this second occurrence on the orbital evolution, however, is negligible. This is because $\dot{M}_{1, \mathrm{ej}}^{\text {self }}$ and $V_{1, t}-V_{1, t}^{*}$ are respectively a factor of about 10 and 6 smaller than the values for the first selfaccretion episode. Neglecting $\mathcal{T}_{\text {self }}$ (short-dashed green curve, Fig. 8d) shows that the relative difference between the post-mass transfer orbital periods with and without self-accretion is about 15 per cent.

As the mass transfer rate decelerates, $\tau_{\text {sync }} / \tau_{\dot{M}}$ correspondingly declines, $\Omega_{1}$ increases back towards synchronous rotation and self-accretion shuts off. Eventually, hydrogen is exhausted in the core, the primary shrinks within its Roche lobe, and mass transfer is terminated (giving rise to the hook feature around $\log _{10}\left(L / L_{\odot}\right) \simeq 2.5$ in Fig. 8a). Mass exchange resumes once the primary re-expands because of $\mathrm{H}$-shell ignition. Since now $\tau_{\text {sync }} / \tau_{\dot{M}} \ll 1$, synchronous rotation is maintained throughout the subsequent mass transfer episode which starts at $t=t_{\mathrm{RLOF}}+5 \times 10^{6} \mathrm{yr}($ Fig. $7 \mathrm{c})$.

Upon He-core ignition, the final mass of the osculating primary is marginally less massive $\left(\approx 0.9 M_{\odot}\right)$ than in the classical models $\left(1.0 M_{\odot}\right)$, the total system mass being held constant. As with the case B model, however, the osculating scheme gives a final orbital period which is a factor of 5.5 smaller $(\approx 6 \mathrm{~d})$ than the classical scheme $(\approx 33$ d, Fig. $8 d)$.

As for the case B model, $\mathcal{F}_{\text {stream }}^{\text {comp }}$ is responsible for the shorter post-mass transfer orbital period. The long-dashed blue curve in Fig. 8 presents the evolution if the $\mathcal{F}_{\text {stream }}$ terms are neglected. When $q>1$, the orbital separation slightly increases (Fig. 8d) because of the dominant, positive contribution from the $\omega r_{\text {acc }} \cos \psi$ term in Eq. (10). The expansion of the orbit reduces the amount that the primary star over-fills its Roche lobe, giving a correspondingly smaller mass loss rate peaking at about $2 \times 10^{-7} M_{\odot} \mathrm{yr}^{-1}$ (Fig. 8c). For a given $t-t_{\mathrm{RLOF}}$, the primary is therefore more massive than found by the other models, and its core-hydrogen burning timescale is, in turn, shorter. By consequence, both shell-hydrogen burning and core-helium burning commence sooner in the binary's evolution and so the duration of mass transfer is shorter. 


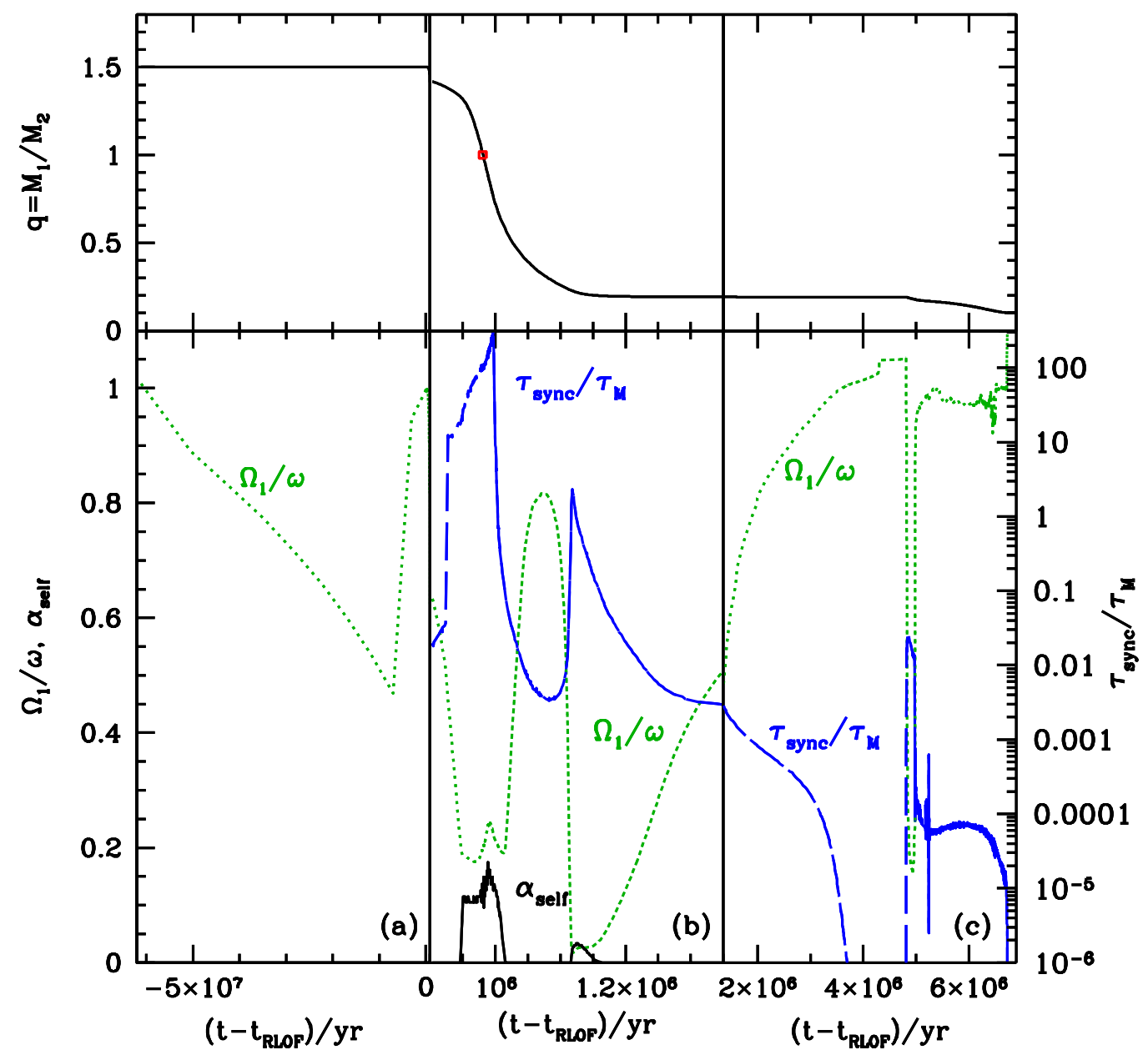

Fig. 7. Similar to Fig. 2, but now for the $6+4 M_{\odot}$ system, $P_{i}=2.5$ days. Here, $t_{\mathrm{RLOF}}=6.12305 \times 10^{7} \mathrm{yr}$.

Eventually, the secondary will fill its Roche lobe as it evolves off the main sequence, before He-core burning has ceased in the primary. This second mass transfer episode starts when the secondary's radius is $17 R_{\odot}$ for the osculating scheme and $53 R_{\odot}$ for the classical model. As for the case B system, we expect common envelope evolution to follow because of the extreme mass ratio.

\section{Discussion}

\subsection{Consequences of the osculating scheme on the orbital evolution}

Our simulations show that the osculating scheme yields a significantly shorter post-mass transfer orbital period than the classical formalism. Alternatively expressed, to obtain an Algol with a given orbital period, the osculating prescription requires a longer initial period. Consequently, the progenitor primary may fill its Roche lobe once it has already developed a deep surface convection zone near the base of the giant branch. Tout \& Eggleton (1988a) suggested this was the case for a number of observed Algols, for example TW Dra and AR Mon. Mass transfer would therefore proceed on the dynamical - rather than the thermal - timescale, possibly causing common envelope evolution. They proposed, however, that such a fate can be avoided if the primary loses sufficient mass via an enhanced stellar wind (companionreinforced attrition process; Tout \& Eggleton 1988b) to reduce the mass ratio close to unity, before RLOF starts.
We currently consider conservative evolution, which may be a reasonable assumption during the slow mass transfer phase. van Rensbergen et al. (2008) suggested that non-conservative mass transfer in Algols is triggered by a hotspot located at the secondary's surface, or at the edge of an accretion disc. They further found that this mechanism typically operates during the rapid mass transfer phase, and becomes quiescent within the slow regime. Other suggested mechanisms - albeit poorly studied - include mass escaping through the outer Lagrangian $\mathcal{L}_{3}$ point (Sytov et al. 2007) or by bipolar jets (Ak et al. 2007). The removal of orbital angular momentum via systemic mass losses was also invoked by De Greve et al. (1985) and De Greve \& Linnell (1994) to explain the observed orbital periods of TV Cas, $\beta$ Lyrae and SV Cen. For TV Cas, for example, the authors estimated that 80 per cent of the transferred mass is ejected from the system, removing about 40 per cent of the progenitor's orbital angular momentum. However, given the effect of $\mathcal{F}_{\text {stream }}$ on the orbital evolution, we argue that a fraction of the estimated orbital angular momentum loss can be attributed to the gravitational interaction between the stars and the mass transfer stream. Therefore, the amount of orbital angular momentum carried by the expelled mass may be lower than quoted by De Greve et al. (1985). We will consider the impact of non-conservative evolution in a future investigation.

Our simulations indicate that, contrarily to what is usually assumed, the primary does not always rotate synchronously throughout mass exchange. As shown in Figs. 2 and 7, the primary is rotating significantly sub-synchronously during the rapid 
P. J. Davis et al.: Binary evolution using the theory of osculating orbits. I.
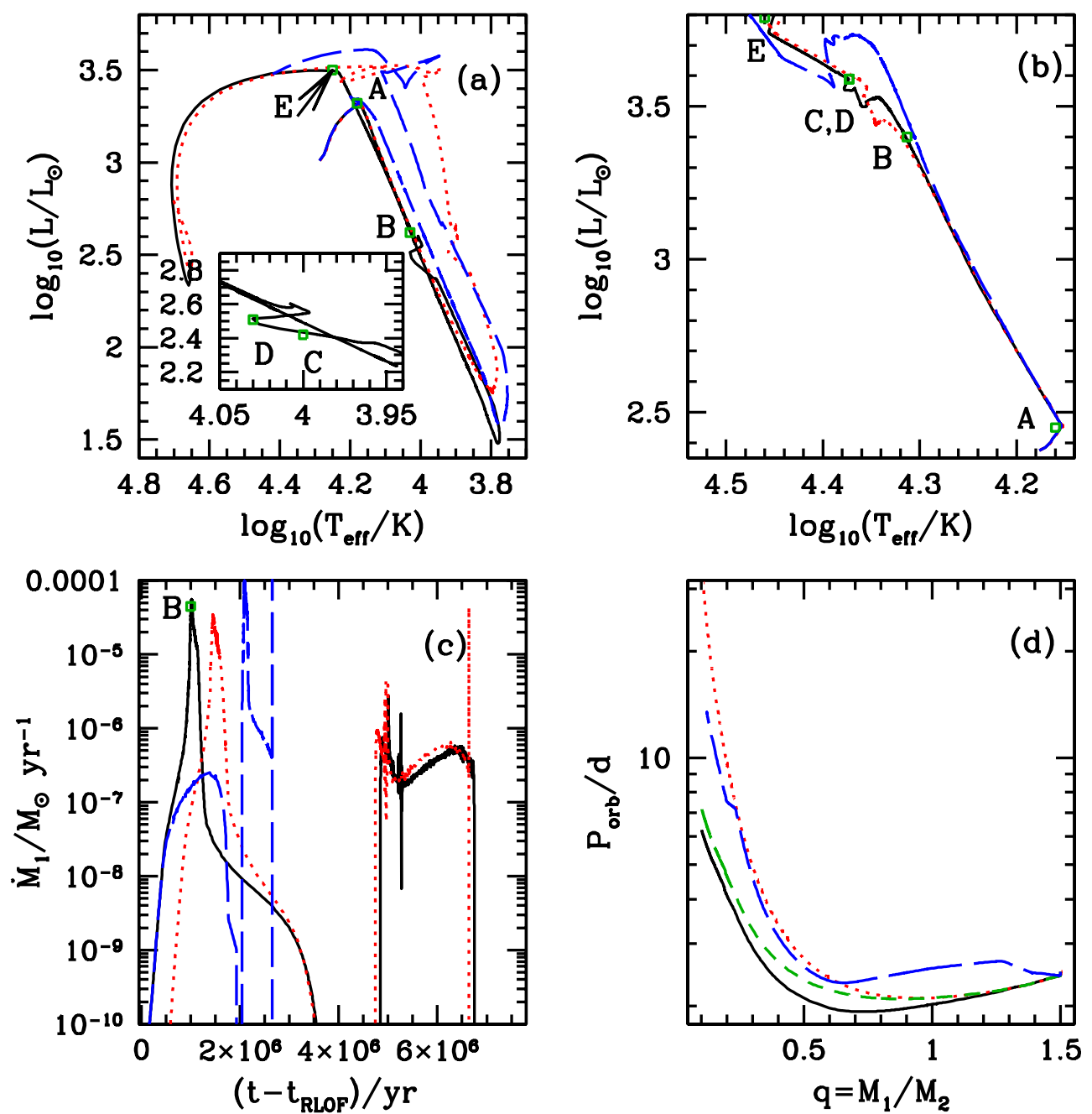

Fig. 8. Similar to Fig. 4 but for the $6+4 M_{\odot}, P_{i}=2.5$ d system. A: start of case A mass transfer; B: $q=1$; C: end of core H-burning; D: start of shell H-burning; E: end of case A mass transfer (H-shell burning ceases).

phase. Only once the mass transfer rate decelerates and convection develops in the surface layers, are tides effective enough to re-synchronize the primary.

Unfortunately, published spin rates of the primary during the rapid phase are, to the best of our knowledge, not available, but this is likely a result of the short duration of this phase (between about $10^{5}$ to $10^{6}$ years in our models). Existing studies of rapid mass transfer systems, such as UX Mon (Sudar et al. 2011), assume that the primary is synchronous, based upon the expectations that tides are always efficient enough to maintain synchronism, although this has never been proven observationally. On the other hand, evidence for synchronous primaries where the mass ratio has reversed are relatively abundant, such as the eclipsing $\delta$ Scuti star KIC 10661783 (Lehmann et al. 2013), TX Uma (Glazunova et al. 2011), KZ Pav (Sürgit et al. 2010) and RX Cas (Andersen et al. 1989).

\subsection{Contact evolution}

The enhanced orbital contraction that self-accretion generates, combined with expanding radiative secondaries in response to mass accretion (e.g. Neo et al. 1977), could lead to more contact systems. The situation may be particularly severe for case A systems when self-accretion operates even after the mass ratio has reversed. Indeed, even though the classical scheme predicts orbital expansion when $q<1$, self-accretion still causes the orbit to contract down to $q \simeq 0.8$ in our case A simulation. However, contact may be avoided if, for a given initial period, the mass ratio is initially close to unity. In this configuration, the mass ratio is reversed sooner, limiting the orbital shrinkage.

The spin-up of the secondary, as it accretes angular momentum from the transferred material (Packet 1981), may also lead to a contact binary. Sepinsky et al. (2007a) demonstrated that the Roche lobe radius of a super-synchronously rotating star will be smaller than the value determined using the standard Eggleton (1983) formula. So, if we also consider the impact of the secondary's rotation on its Roche radius (as should be done), we indeed find that all our models enter a contact configuration during the rapid phase. However, magnetic braking may prevent significant spin-up of the secondary (Dervişoğlu et al. 2010; Deschamps et al. 2013) although, in light of the expanding radiative secondary, these mechanisms may only delay the onset of contact.

Contact evolution requires detailed modelling of energy transport between the stars within their common envelope (e.g. Webbink 1977b; Stępien 2009), which is beyond the scope of the present paper. Nonetheless, observations of the contact systems with early spectral-type stars, such as LY Aur (Zhao et al. 2014), V382 Cyg and TU Mus (Qian et al. 2007) indicate that their 
evolution is similar to that of a semi-detached Algol, but much shorter-lived. The authors suggest that the contact configuration was most likely triggered during a rapid case A mass transfer phase, and that the observed period increase is caused by mass transfer from the less massive to the more massive star. They also expect that the increasing orbital separation will break the contact configuration, giving a semi-detached Algol, suggesting that not all contact systems necessarily merge.

Further complications arise from the fact that the rotation rate of each star is different. The concept of the Roche model, which assumes that both stars rotate synchronously with the orbit, is therefore incorrect. As outlined by Vanbeveren (1977), asynchronous rotation of both components gives Roche lobes that do not necessarily coincide at a common inner-Lagrangian point. This situation may greatly complicate the mass flow structure between the stars, possibly leading to non-conservative evolution. We appeal to smooth particle hydrodynamical (SPH) simulations to investigate this in further detail.

\subsection{Physical considerations}

\subsubsection{Stellar rotation}

By adopting the solid-body approximation, we assume that torques will spin up or spin down each star as a whole. However, it is more likely that only the outer-most layers will be affected, thereby triggering differential rotation. Subsequently, angular momentum is re-distributed within the stellar interior via meridional circulation and shear instabilities (see Maeder 2009, for a review).

Song et al. (2013) studied the affect of angular momentum transport within a differentially rotating $15 M_{\odot}$ primary, with a $10 M_{\odot}$ companion as the primary evolved from the ZAMS until the onset of RLOF. They found that meridional circulation always counteracts the impact of tides; spinning up the surface layers when tides spin them down and vice versa, increasing the time for the star to rotate synchronously with the orbit. Consequently, some of their models commence RLOF before the primary is synchronised. In addition, differential rotation significantly enhances nitrogen abundances at the stellar surface.

We can speculate that significant differential rotation will occur while our donors become sub-synchronous, with meridional circulation initially opposing the spin-down triggered by rapid mass loss, and then the subsequent tidal forces which act to resynchronise the donor. In turn, this may affect both the duration of the self-accretion phase and the value of $\alpha_{\text {self }}$.

\subsubsection{Tides}

Tassoul $(1987,1988)$ proposed an alternative theory that invokes large-scale hydrodynamical flows as a means to dissipate kinetic energy, which are more efficient than the dynamical tide model described by Zahn (1977). Indeed, the synchronization and circularisation timescales between the two approaches vary by up to three orders of magnitude (Khaliullin \& Khaliullina 2010).

We can speculate on the impact of Tassoul's formalism on our calculations as follows. If $\tau_{\text {sync }}$ is 1000 times shorter, then for our case B system $\tau_{\text {sync }}<\tau_{R}$, i.e. tides keep the donor in synchronous rotation by the time RLOF starts. Additionally, for both the case A and case B models, an inspection of Figs. 2 and 7 shows that we would have $\tau_{\text {sync }}<\tau_{\dot{M}}$. Hence, even during the fast phase, mass loss would not be sufficiently rapid to spin down the donor star to sub-synchronous rates and, in turn, self-accretion would not be triggered. In this case, the orbital evolution will correspond to the short-dashed green curves given in Figs. 4 and 8, where we neglected the $\mathcal{T}_{\text {self }}$ term. Therefore, using Tassoul's formalism will not significantly affect our results, namely that our osculating scheme still yields much shorter orbital periods.

However, the hydrodynamical model has since been criticised on theoretical grounds by Rieutord (1992) and Rieutord \& Zahn (1997; but see Tassoul \& Tassoul 1997, for a counterargument), while observations attempting to constrain the mechanism underpinning tidal interactions are inconclusive. On the one hand, Claret et al. (1995) and Claret \& Cunha (1997) found that both the Zahn and Tassoul formalisms can adequately account for the observed eccentricities and rotational velocities of early-type eclipsing binaries. On the other hand, using an updated sample of such binaries, Khaliullin \& Khaliullina (2007, 2010) found that Tassoul's theory is in contradiction with observations, which are better reproduced by Zahn's formalism. However, Meibom \& Mathieu (2005) derived, for stellar populations with a range of ages, the tidal circularization period (i.e. the orbital period at which the binary orbit circularises at the age of the population). Their results indicated that, at a given age, the observed circularisation period is larger than the predicted value from the dynamical tide model, suggesting that it is too inefficient. Clearly, more observational and theoretical work is required in this area.

\subsubsection{Self-accretion}

The phenomenon of self-accretion has also been found in the studies of Kruszewski (1964a) and Sepinsky et al. (2010), who use the same ballistic approach, which assumes that no collisions between particles occur. Clearly, this is not realistic, as there are indeed multiple collisions along the trajectory.

Nonetheless, our results are in qualitative agreement with the SPH calculations of Belvedere et al. (1993), who find that below some critical rotational velocity, the entire stream is deflected towards the primary. For larger spin rates, material falls onto both components. However, their study focused on the impact of asynchronous rotation on disc formation around the secondary, and they did not quantify self-accretion. We hope our work will motivate future SPH studies in this area.

\section{Summary and conclusions}

We use our stellar binary evolution code BINSTAR to calculate the evolution of Algol systems using the theory of osculating orbital elements. By calculating the ballistic trajectories of ejected particles from the mass losing star (the donor), we determine the change of linear momentum of each star, and the gravitational perturbation applied to the stars by the mass transfer stream. As a consequence of the latter, the osculating formalism predicts signicantly shorter post-mass transfer orbital periods, typically by a factor of 4 , than the widely applied classical scheme.

Also contrary to widely held belief, the donor star does not remain in synchronous rotation with the orbital motion throughout mass exchange. The initially rapid mass ejection spins down the donor on a shorter timescale than the tidal synchronization timescale, enforcing sub-synchronous rotation and causing about 15 to 20 per cent of the ejected material to fall back onto the donor during these episodes of self-accretion. Self-accretion, combined with the sink of orbital angular momentum that mass transfer provides, may lead to the formation of more contact binary systems. 
P. J. Davis et al.: Binary evolution using the theory of osculating orbits. I.

While we have mainly focused on conservative Algol evolution, the osculating prescription clearly applies to all varieties of interacting binaries. In the future, we will apply our osculating formalism to investigate the evolution of eccentric systems.

Acknowledgements. P.J.D. acknowledges financial support from the FNRS Research Fellowship - Chargé de Recherche. L.S. is a FNRS Research Associate. We thank the anonymous referee whose constructive comments helped to improve the quality of the manuscript.

\section{References}

Ak, H., Chadima, P., Harmanec, P., et al. 2007, A\&A, 463, 233 Andersen, J., Pavlovski, K., \& Piirola, V. 1989, A\&A, 215, 272 Andersen, J., Nordstrom, B., \& Clausen, J. V. 1990, A\&A, 228, 365 Belvedere, G., Lanzafame, G., \& Molteni, D. 1993, A\&A, 280, 525 Bonačić Marinović, A. A., Glebbeek, E., \& Pols, O. R. 2008, A\&A, 480, 797 Chen, X., Han, Z., Deca, J., \& Podsiadlowski, P. 2013, MNRAS, accepted [arXiv: 1306.3281$]$

Claret, A., \& Cunha, N. C. S. 1997, A\&A, 318, 187

Claret, A., Gimenez, A., \& Cunha, N. C. S. 1995, A\&A, 299, 724

Davis, P. J., Siess, L., \& Deschamps, R. 2013, A\&A, 556, A4

De Greve, J. P., \& Linnell, A. P. 1994, A\&A, 291, 786

De Greve, J. P., Packet, W., \& de Landtsheer, A. C. 1985, A\&A, 142, 367

Dervişoğlu, A., Tout, C. A., \& Ibanoğlu, C. 2010, MNRAS, 406, 1071

Deschamps, R., Siess, L., Davis, P. J., \& Jorissen, A. 2013, A\&A, 557, A40

Edwards, D. A., \& Pringle, J. E. 1987, MNRAS, 229, 383

Eggleton, P. P. 1983, ApJ, 268, 368

Flannery, B. P. 1975, MNRAS, 170, 325

Geller, A. M., \& Mathieu, R. D. 2011, Nature, 478, 356

Glazunova, L. V., Mkrtichian, D. E., \& Rostopchin, S. I. 2011, MNRAS, 415, 2238

Gokhale, V., Peng, X. M., \& Frank, J. 2007, ApJ, 655, 1010

Habets, G. M. H. J., \& Zwaan, C. 1989, A\&A, 211, 56

Hadjidemetriou, J. D. 1969a, Ap\&SS, 3, 330

Hadjidemetriou, J. D. 1969b, Ap\&SS, 3, 31

Han, Z., Podsiadlowski, P., Maxted, P. F. L., Marsh, T. R., \& Ivanova, N. 2002, MNRAS, 336, 449

Hoyle, F., \& Fowler, W. A. 1960, ApJ, 132, 565

Khaliullin, K. F., \& Khaliullina, A. I. 2007, MNRAS, 382, 356

Khaliullin, K. F., \& Khaliullina, A. I. 2010, MNRAS, 401, 257

Kolb, U., \& Ritter, H. 1990, A\&A, 236, 385

Kruszewski, A. 1964a, Acta Astron., 14, 231

Kruszewski, A. 1964b, Acta Astron., 14, 241

Lehmann, H., Southworth, J., Tkachenko, A., \& Pavlovski, K. 2013, A\&A, 557, A79

Leigh, N., Knigge, C., Sills, A., et al. 2013, MNRAS, 428, 897

Limber, D. N. 1963, ApJ, 138, 1112

Lubow, S. H., \& Shu, F. H. 1975, ApJ, 198, 383

Luk’yanov, L. G. 2008, Astron. Rep., 52, 680
Maeder, A. 2009, Physics, Formation and Evolution of Rotating Stars (Berlin, Heidelberg: Springer)

McCrea, W. H. 1964, MNRAS, 128, 147

Meibom, S., \& Mathieu, R. D. 2005, ApJ, 620, 970

Meibom, S., Mathieu, R. D., \& Stassun, K. G. 2006, ApJ, 653, 621

Mengel, J. G., Norris, J., \& Gross, P. G. 1976, ApJ, 204, 488

Neo, S., Miyaji, S., Nomoto, K., \& Sugimoto, D. 1977, PASJ, 29, 249

Packet, W. 1981, A\&A, 102, 17

Paczyński, B. 1971, ARA\&A, 9, 183

Paczynski, B. 1976, in Structure and Evolution of Close Binary Systems, eds. P. Eggleton, S. Mitton, \& J. Whelan, IAU Symp., 73, 75

Piotrowski, S. L. 1964, Acta Astron., 14, 251

Piotrowski, S. L. 1967, Sov. Astron., 11, 191

Pratt, J. P., \& Strittmatter, P. A. 1976, ApJ, 204, L29

Pringle, J. E., \& Wade, R. A. 1985, Interacting binary stars (Cambridge University Press)

Qian, S.-B., Yuan, J.-Z., Liu, L., et al. 2007, MNRAS, 380, 1599

Raymer, E. 2012, MNRAS, 427, 1702

Rieutord, M. 1992, A\&A, 259, 581

Rieutord, M., \& Zahn, J.-P. 1997, ApJ, 474, 760

Savonije, G. J. 1978, A\&A, 62, 317

Sepinsky, J. F., Willems, B., \& Kalogera, V. 2007a, ApJ, 660, 1624

Sepinsky, J. F., Willems, B., Kalogera, V., \& Rasio, F. A. 2007b, ApJ, 667, 1170

Sepinsky, J. F., Willems, B., Kalogera, V., \& Rasio, F. A. 2010, ApJ, 724, 546

Siess, L. 2010, A\&A, 512, A10

Siess, L., Izzard, R. G., Davis, P. J., \& Deschamps, R. 2013, A\&A, 550, A100

Song, H. F., Maeder, A., Meynet, G., et al. 2013, A\&A, 556, A100

Stępień, K. 2009, MNRAS, 397, 857

Sterne, T. E. 1960, An introduction to celestial mechanics (New York: Interscience Publication)

Sudar, D., Harmanec, P., Lehmann, H., et al. 2011, A\&A, 528, A146

Sürgit, D., Erdem, A., \& Budding, E. 2010, MNRAS, 407, 497

Sytov, A. Y., Kaigorodov, P. V., Bisikalo, D. V., Kuznetsov, O. A., \& Boyarchuk, A. A. 2007, Astron. Rep., 51, 836

Tassoul, J.-L. 1987, ApJ, 322, 856

Tassoul, J.-L. 1988, ApJ, 324, L71

Tassoul, M., \& Tassoul, J.-L. 1997, ApJ, 481, 363

Tout, C. A., \& Eggleton, P. P. 1988a, ApJ, 334, 357

Tout, C. A., \& Eggleton, P. P. 1988b, MNRAS, 231, 823

Ulrich, R. K., \& Burger, H. L. 1976, ApJ, 206, 509

van Rensbergen, W., De Greve, J. P., De Loore, C., \& Mennekens, N. 2008, A\&A, 487, 1129

Vanbeveren, D. 1977, A\&A, 54, 877

Wang, B., \& Han, Z. 2012, New Astron. Rev., 56, 122

Webbink, R. F. 1977a, ApJ, 211, 486

Webbink, R. F. 1977b, ApJ, 215, 851

Webbink, R. F. 2008, in Astrophys. Space Sci. Lib. 352, eds. E. F. Milone, D. A. Leahy, \& D. W. Hobill, 233

Yakut, K., Aerts, C., \& Morel, T. 2007, A\&A, 467, 647

Zahn, J.-P. 1977, A\&A, 57, 383

Zahn, J.-P. 1989, A\&A, 220, 112

Zeldovich, Y. B., \& Guseynov, O. H. 1966, ApJ, 144, 840

Zhao, E., Qian, S., Li, L., et al. 2014, New Astron., 26, 112

Pages 14 to 16 are available in the electronic edition of the journal at http://wWw . aanda. org 


\section{Appendix A: The perturbing forces $\mathcal{S}_{\text {self }}$ and $\mathcal{T}_{\text {self }}$}

Consider a primary star of mass $M_{1}$. At time $t=t_{0}$, it has an orbital velocity $\boldsymbol{v}_{1}=\boldsymbol{v}_{0}$, and an orbital angular velocity $\omega$. At a later time $t=t^{\prime}$, a particle is ejected from the primary at the inner-Lagrangian point, located at a distance $r_{\mathcal{L}_{1}}$ from the primary's mass centre (see Fig. A.1), and so the primary's mass becomes $M_{1}+\delta M_{1, \mathrm{ej}}^{\text {self }}$, where $\delta M_{1, \mathrm{ej}}^{\text {self }}<0$. The particle's absolute velocity is $\boldsymbol{W}_{\mathrm{ej}}^{\prime}$. As a result, the centre of mass is shifted by

$\delta \boldsymbol{r}_{1}=\frac{\delta M_{1, \mathrm{ej}}^{\text {self }}}{M_{1}} \boldsymbol{r}_{\mathcal{L}_{1}}$

with respect to its unperturbed location, and its new orbital velocity is $\boldsymbol{v}_{1}^{\prime}$. During a time interval $\delta t$, the change in the primary's orbital velocity is (see Hadjidemetriou 1969b; Sepinsky et al. $2007 \mathrm{~b}$, for further details)

$$
\begin{aligned}
\frac{\boldsymbol{v}_{1}^{\prime}-\boldsymbol{v}_{1}}{\delta t}= & \frac{\boldsymbol{Q}_{1}^{\prime}-\boldsymbol{Q}_{1}}{\delta t} \frac{1}{M_{1}}+\frac{\delta M_{1, \mathrm{ej}}^{\text {self }}}{\delta t} \frac{1}{M_{1}}\left(\boldsymbol{V}_{\mathrm{ej}}+\boldsymbol{\omega}^{\prime} \times \boldsymbol{r}_{\mathcal{L}_{1}}\right) \\
& +\frac{1}{M_{1}} \frac{\delta M_{1, \mathrm{ej}}^{\text {self }}}{(\delta t)^{2}} \boldsymbol{r}_{\mathcal{L}_{1}},
\end{aligned}
$$

where $\boldsymbol{Q}_{1}$ is the primary's momentum, primed quantities indicate values at time $t^{\prime}$, and $\boldsymbol{V}_{\mathrm{ej}}=\boldsymbol{W}_{\mathrm{ej}}-\boldsymbol{v}_{1}$ is the relative velocity of the ejected material with respect to the primary's mass centre.

At self-accretion, the primary accretes the particle of mass $\delta M_{1, \text { acc }}^{\text {self }}=-\delta M_{1, \text { ej }}^{\text {self }}>0$. Just before self-accretion occurs at time $t=t^{\prime \prime}$, the momentum of the primary and ejected particle, $Q_{1}^{\prime \prime}$, is

$\boldsymbol{Q}_{1}^{\prime \prime}=\left(M_{1}-\delta M_{1, \text { acc }}^{\text {self }}\right) \boldsymbol{v}_{1}^{\prime \prime}+\delta M_{1, \text { acc }}^{\text {self }} \boldsymbol{W}_{\text {acc }}$

where $\boldsymbol{W}_{\text {acc }}$ is the absolute velocity of the self-accreted particle. The orbital velocity, $\boldsymbol{v}_{1}^{\prime \prime}$, is the sum of the non-perturbed orbital velocity at time $t^{\prime \prime}, \boldsymbol{v}_{0}^{\prime \prime}$, and the perturbation to the velocity because of the original ejection episode, so

$\boldsymbol{v}_{1}^{\prime \prime}=\boldsymbol{v}_{0}^{\prime \prime}+\left(\omega^{\prime}+\delta \omega\right) \times \delta \boldsymbol{r}_{1}=\boldsymbol{v}_{0}^{\prime \prime}+\frac{\delta M_{1, \mathrm{ej}}^{\text {self }}}{M_{1}}\left(\omega^{\prime} \times \boldsymbol{r}_{\mathcal{L}_{1}}\right)$,

where we have used Eq. (A.1) and ignored terms larger than firstorder. Inserting Eq. (A.4) into Eq. (A.3) gives

$\boldsymbol{Q}_{1}^{\prime \prime}=M_{1} \boldsymbol{v}_{0}^{\prime \prime}+\delta M_{1, \mathrm{ej}}^{\text {self }}\left(\omega^{\prime} \times \boldsymbol{r}_{\mathcal{L}_{1}}\right)-\delta M_{1, \mathrm{acc}}^{\text {self }} \boldsymbol{v}_{0}^{\prime \prime}+\delta M_{1, \mathrm{acc}}^{\text {self }} \boldsymbol{W}_{\mathrm{acc}}$.

The particle is self-accreted at time $t^{\prime \prime \prime}$, at a location $\boldsymbol{r}_{\text {acc }}^{*}$ with respect to the primary's mass centre. This shifts the mass centre by an amount

$\delta \boldsymbol{r}_{\mathrm{self}}=\frac{\delta M_{1, \mathrm{acc}}^{\text {self }}}{M_{1}} \boldsymbol{r}_{\mathrm{acc}}^{*}$

and the primary's momentum is now

$Q^{\prime \prime \prime}=M_{1} v_{1}^{\prime \prime \prime}$.

The new orbital velocity, $\boldsymbol{v}_{1}^{\prime \prime \prime}$, is the sum of the unperturbed orbital velocity, $v_{0}^{\prime \prime \prime}$, and the perturbations to the velocity arising from the ejection and self-accretion processes, i.e.

$$
\begin{aligned}
\boldsymbol{v}_{1}^{\prime \prime \prime} & =\boldsymbol{v}_{0}^{\prime \prime \prime}+\frac{\delta M_{1, \mathrm{ej}}^{\text {self }}}{M_{1}}\left(\omega^{\prime} \times \boldsymbol{r}_{\mathcal{L}_{1}}\right)+\left(\boldsymbol{\omega}^{\prime \prime}+\delta \omega\right) \times \delta \boldsymbol{r}_{\text {self }} \\
& =\boldsymbol{v}_{0}^{\prime \prime \prime}+\frac{\delta M_{1, \mathrm{ej}}^{\text {self }}}{M_{1}}\left(\omega^{\prime} \times \boldsymbol{r}_{\mathcal{L}_{1}}\right)+\frac{\delta M_{1, \mathrm{acc}}^{\text {self }}}{M_{1}}\left(\omega^{\prime \prime} \times \boldsymbol{r}_{\mathrm{acc}}^{*}\right)
\end{aligned}
$$

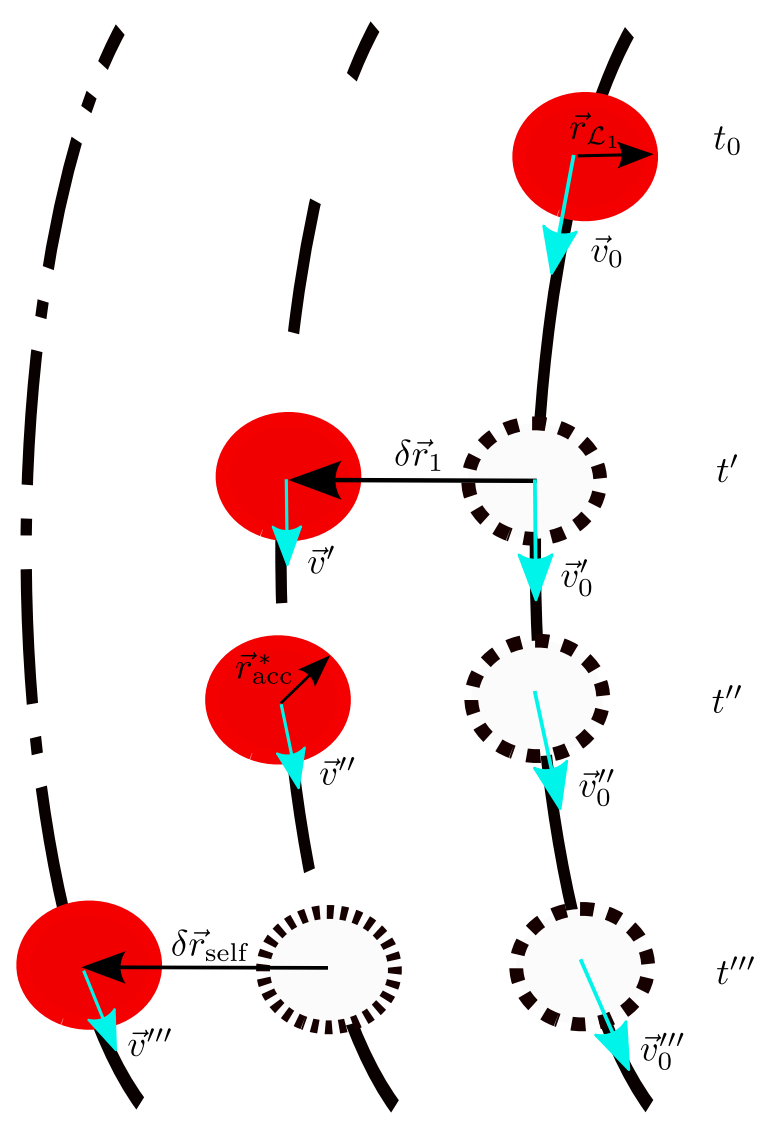

Fig. A.1. Illustration of the self-accretion process. At time $t=t_{0}$, the donor star moves along its orbital path (solid black curve), with an orbital velocity $\boldsymbol{v}_{0}$ (cyan arrow). At $t^{\prime}$, a particle of mass $\delta M_{1, \mathrm{ej}}^{\text {self }}$ is ejected from the inner-Lagrangian point located at $\boldsymbol{r}_{\mathcal{L}_{1}}$ with respect to the donor's mass centre. The ejection shifts the centre of mass by $\delta \boldsymbol{r}_{1}$, and the donor follows a new orbit (long dashed curve) with velocity $\boldsymbol{v}^{\prime}$. At $t^{\prime \prime}$ just before the particle is re-accreted (at $\boldsymbol{r}_{\text {acc }}^{*}$ ), the orbital velocity is $\boldsymbol{v}^{\prime \prime}$. Subsequently, self-accretion shifts the donor's mass centre by $\delta \boldsymbol{r}_{\text {self }}$, and it follows the orbit indicated by the dot-dashed curve, with a velocity $\boldsymbol{v}^{\prime \prime \prime}$. The dashed circles represent the locations of the donor if no mass ejection had taken place, while the dotted circle indicates the donor's location had no self-accretion occurred.

where we have used Eq. (A.6), and once again ignored terms higher than first-order. Inserting Eq. (A.8) into Eq. (A.7) yields

$$
\boldsymbol{Q}_{1}^{\prime \prime \prime}=M_{1} \boldsymbol{v}_{0}^{\prime \prime \prime}+\delta M_{1, \mathrm{ej}}^{\text {self }}\left(\omega^{\prime} \times \boldsymbol{r}_{\mathcal{L}_{1}}\right)+\delta M_{1, \mathrm{acc}}^{\text {self }}\left(\omega^{\prime \prime} \times \boldsymbol{r}_{\mathrm{acc}}^{*}\right) .
$$

Taking the difference between Eqs. (A.9) and (A.5), dividing the result by $\delta t$ and using the fact that $\delta M_{1, \mathrm{ej}}^{\text {self }}=-\delta M_{1, \text { acc }}^{\text {self }}$ yields

$$
\begin{aligned}
\frac{\boldsymbol{v}_{0}^{\prime \prime \prime}-\boldsymbol{v}_{0}^{\prime \prime}}{\delta t}= & \frac{1}{M_{1}} \frac{\boldsymbol{Q}_{1}^{\prime \prime \prime}-\boldsymbol{Q}_{1}^{\prime \prime}}{\delta t}-\frac{\delta M_{1, \mathrm{acc}}^{\text {self }}}{\delta t} \frac{1}{M_{1}}\left(\boldsymbol{\omega}^{\prime \prime} \times \boldsymbol{r}_{\mathrm{acc}}^{*}\right) \\
& -\frac{\delta M_{1, \mathrm{acc}}^{\mathrm{self}}}{\delta t} \frac{1}{M_{1}} \boldsymbol{v}_{0}^{\prime \prime}+\frac{\delta M_{1, \mathrm{acc}}^{\mathrm{self}}}{\delta t} \frac{1}{M_{1}} \boldsymbol{W}_{\mathrm{acc}}
\end{aligned}
$$

Following Sepinsky et al. (2007b), the absolute acceleration of the primary's mass centre is the sum of Eq. (A.10), the relative acceleration of the primary's mass centre, $\frac{\dot{M}_{1, \text { acc }}^{\text {self }}}{(\delta t)^{2}} \frac{1}{M_{1}}$, and the 
P. J. Davis et al.: Binary evolution using the theory of osculating orbits. I.

Coriolis acceleration, $\frac{1}{M_{1}} \frac{\delta M_{1, \text { acc }}^{\text {self }}}{\delta t} 2\left(\omega^{\prime \prime} \times \boldsymbol{r}_{\mathrm{acc}}^{*}\right)$ to give

$$
\begin{aligned}
\frac{\boldsymbol{v}_{1}^{\prime \prime \prime}-\boldsymbol{v}_{1}^{\prime \prime}}{\delta t}= & \frac{1}{M_{1}} \frac{\boldsymbol{Q}_{1}^{\prime \prime \prime}-\boldsymbol{Q}_{1}^{\prime \prime}}{\delta t}+\frac{\delta M_{1, \mathrm{acc}}^{\text {self }}}{\delta t} \frac{1}{M_{1}}\left[\left(\boldsymbol{\omega}^{\prime \prime} \times \boldsymbol{r}_{\mathrm{acc}}^{*}\right)+\boldsymbol{V}_{\mathrm{acc}}\right] \\
& +\frac{1}{M_{1}} \frac{\delta M_{1, \mathrm{acc}}^{\mathrm{self}}}{(\delta t)^{2}}
\end{aligned}
$$

where $\boldsymbol{V}_{\text {acc }}=\boldsymbol{W}_{\text {acc }}-\boldsymbol{v}_{0}^{\prime \prime}$ is the relative velocity of the self-accreted particle with respect to the primary's mass centre.

Summing Eqs. (A.11) and (A.2) gives the acceleration of the primary's mass centre from both the ejection and self-accretion process. In the limit $\delta t \rightarrow 0$, and remembering that $\delta M_{1, \text { acc }}^{\text {self }}=$ $-\delta M_{1, \mathrm{ej}}^{\text {self }}$, then the acceleration of the primary's mass centre is

$$
\begin{aligned}
\frac{\mathrm{d}^{2} \boldsymbol{R}_{1}}{\mathrm{~d} t^{2}}= & \frac{\boldsymbol{F}_{1}}{M_{1}}+\frac{\dot{M}_{1, \mathrm{ej}}^{\text {self }}}{M_{1}}\left[\left(\boldsymbol{V}_{\mathrm{ej}}-\boldsymbol{V}_{\mathrm{acc}}\right)+\left(\boldsymbol{\omega}^{\prime} \times \boldsymbol{r}_{\mathcal{L}_{1}}-\boldsymbol{\omega}^{\prime \prime} \times \boldsymbol{r}_{\mathrm{acc}}^{*}\right)\right] \\
& +\frac{\ddot{M}_{1, \mathrm{ej}}^{\text {self }}}{M_{1}}\left(\boldsymbol{r}_{\mathcal{L}_{1}}-\boldsymbol{r}_{\mathrm{acc}}^{*}\right),
\end{aligned}
$$

where $\boldsymbol{R}_{1}$ is the position vector of the primary with respect to an inertial reference frame, and $\boldsymbol{F}_{1}$ is the sum of all external forces acting on the primary, which writes as

$\boldsymbol{F}_{1}=-\frac{G M_{1} M_{2}}{r^{2}} \frac{\boldsymbol{R}_{1}}{R_{1}}+\boldsymbol{f}_{1}$,

where $\boldsymbol{r}=\boldsymbol{R}_{2}-\boldsymbol{R}_{1}, \boldsymbol{R}_{2}$ is the position vector of the secondary, and $\boldsymbol{f}_{1}$ is the force acting on the primary via the matter stream. Since the secondary is not accreting, its equation of motion is

$\frac{\mathrm{d}^{2} \boldsymbol{R}_{2}}{\mathrm{~d} t^{2}}=-\frac{G M_{1}}{r^{2}} \frac{\boldsymbol{R}_{2}}{R_{2}}+\frac{\boldsymbol{f}_{2}}{M_{2}}$,

where $f_{2}$ is the force acting on the secondary by the accretion stream. Subtracting Eq. (A.12) from Eq. (A.14) gives the equation of motion of the secondary with respect to the primary, which is

$$
\begin{aligned}
\frac{\mathrm{d}^{2} \boldsymbol{r}}{\mathrm{d} t^{2}}= & -\frac{G\left(M_{1}+M_{2}\right)}{r^{3}} \boldsymbol{r}+\frac{\boldsymbol{f}_{2}}{M_{2}}-\frac{\boldsymbol{f}_{1}}{M_{1}}-\frac{\dot{M}_{1, \mathrm{ej}}^{\text {self }}}{M_{1}}\left[\left(\boldsymbol{V}_{\mathrm{ej}}-\boldsymbol{V}_{\mathrm{acc}}\right)\right. \\
& \left.+\left(\omega^{\prime} \times \boldsymbol{r}_{\mathcal{L}_{1}}-\omega^{\prime \prime} \times \boldsymbol{r}_{\mathrm{acc}}^{*}\right)\right]-\frac{\ddot{M}_{1, \mathrm{ej}}^{\text {self }}}{M_{1}}\left(\boldsymbol{r}_{\mathcal{L}_{1}}-\boldsymbol{r}_{\mathrm{acc}}^{*}\right),
\end{aligned}
$$

which takes the form

$\frac{\mathrm{d}^{2} \boldsymbol{r}}{\mathrm{d} t^{2}}=-\frac{G\left(M_{1}+M_{2}\right)}{r^{3}} \boldsymbol{r}+\mathcal{S}_{\text {self }} \hat{e}_{r}+\mathcal{T}_{\text {self }} \hat{e}_{t}$.

Here, $\hat{e}_{r}$ is a unit vector pointing along $\boldsymbol{r}$, and $\hat{e}_{t}$ is a unit vector perpendicular to $\hat{e}_{r}$ in the direction of the orbital motion. Taking the dot product of Eq. (A.16) with $\hat{e}_{r}$ and $\hat{e}_{t}$ respectively, yields

$$
\begin{aligned}
\mathcal{S}_{\text {self }}= & \frac{f_{2, r}}{M_{2}}-\frac{f_{1, r}}{M_{1}}-\frac{\ddot{M}_{1, \mathrm{ej}}^{\text {self }}}{M_{1}}\left(r_{\mathcal{L}_{1}}-r_{\mathrm{acc}}^{*} \cos \psi^{*}\right) \\
& -\frac{\dot{M}_{1, \mathrm{ej}}^{\text {self }}}{M_{1}}\left(V_{\mathrm{ej}, r}-V_{\mathrm{acc}, r}+\omega^{\prime \prime} r_{\mathrm{acc}}^{*} \sin \psi^{*}\right),
\end{aligned}
$$

and

$$
\begin{aligned}
\mathcal{T}_{\text {self }}= & \frac{f_{2, t}}{M_{2}}-\frac{f_{1, t}}{M_{1}}-\frac{\ddot{M}_{1, \mathrm{ej}}^{\text {self }}}{M_{1}} r_{\mathrm{acc}}^{*} \sin \psi^{*} \\
& -\frac{\dot{M}_{1, \mathrm{ej}}^{\text {self }}}{M_{1}}\left(V_{\mathrm{ej}, t}-V_{\mathrm{acc}, t}+\omega^{\prime} r_{\mathcal{L}_{1}}-\omega^{\prime \prime} r_{\mathrm{acc}}^{*} \cos \psi^{*}\right),
\end{aligned}
$$

which are the same as Eqs. (11) and (12), noting that $\omega^{\prime}=\omega^{\prime \prime}=$ $\omega$ for circular orbits. The quantity $\psi^{*}$ is the angle between $\hat{e}_{r}$ and the impact site, and the subscripts " $r$ " and " $t$ " indicate components along $\hat{e}_{r}$ and $\hat{e}_{t}$ respectively.

\section{Appendix B: Torque arising from mass transfer, $\dot{J}_{\mathrm{MT}}$}

Consider a primary star of mass $M_{1}$, and a secondary of mass $M_{2}$, separated by a distance $r$. They respectively orbit the common centre of mass with an orbital velocity $\boldsymbol{v}_{1}$ and $\boldsymbol{v}_{2}$. The velocity of the secondary with respect to the primary is $\boldsymbol{v}=\boldsymbol{v}_{2}-\boldsymbol{v}_{1}$, and the orbital angular momentum is given by

$J_{\text {orb }}=m\left[G M a\left(1-e^{2}\right)\right]^{1 / 2}=m r v_{t}$,

where $e$ is the eccentricity, $m=M_{1} M_{2} / M$ is the reduced mass, $M=M_{1}+M_{2}$, and $v_{t}$ is the orbital velocity along $\hat{e}_{t}$, given by

$v_{t}=\left[\frac{G M}{a\left(1-e^{2}\right)}\right]^{1 / 2}(1+e \cos v)$

and $v$ is the true anomaly. Taking the time derivative of the last equality in Eq. (B.1) and noting that $\dot{r}=0$ for an osculating orbit (see, e.g. Bonačić Marinović et al. 2008), yields

$\frac{\dot{J}_{\text {orb }}}{J_{\text {orb }}}=\frac{\dot{M}_{1}}{M_{1}}+\frac{\dot{M}_{2}}{M_{2}}-\frac{\dot{M}}{M}+\frac{\dot{v}_{t}}{v_{t}}$.

Similarly to Eq. (A.16), the equation of motion of a binary acted on by perturbing forces $\mathcal{S}$ and $\mathcal{T}$ reads

$\frac{\mathrm{d}^{2} \boldsymbol{r}}{\mathrm{d} t^{2}}=\frac{\mathrm{d} \boldsymbol{v}}{\mathrm{d} t}=-\frac{G\left(M_{1}+M_{2}\right)}{|r|^{3}} \boldsymbol{r}+\mathcal{S} \hat{e}_{r}+\mathcal{T} \hat{e}_{t}$.

Taking the dot product of Eq. (B.4) with $\hat{e}_{t}$ gives

$\frac{\mathrm{d} v_{t}}{\mathrm{~d} t}=\mathcal{T}$

Inserting Eqs. (B.5) and (B.2) into Eq. (B.3), and using the first equality in Eq. (B.1), gives the torque applied onto the orbit from mass transfer,

$\dot{J}_{\text {orb,MT }}=J_{\text {orb }}\left(\frac{\dot{M}_{1}}{M_{1}}+\frac{\dot{M}_{2}}{M_{2}}-\frac{\dot{M}}{M}\right)+m \frac{a\left(1-e^{2}\right)}{1+e \cos v} \mathcal{T}$.

The net change of the primary's mass is the sum of mass transferred to the companion via RLOF, $\dot{M}_{1, \mathrm{ej}}^{\text {comp }}<0$, and mass ejected by the wind, $\dot{M}_{1, \text { loss }}<0$, i.e.

$\dot{M}_{1}=\dot{M}_{1, \text { ej }}^{\text {comp }}+\dot{M}_{1, \text { loss }}$.

Similarly, for the secondary

$\dot{M}_{2}=-\beta \dot{M}_{1, \mathrm{ej}}^{\mathrm{comp}}+\dot{M}_{2, \mathrm{loss}}$,

where the first term on the right hand side gives the accretion rate and $\dot{M}_{2, \text { loss }}$ includes the mass ejected from the system during non-conservative mass transfer. Substituting Eqs. (B.7) and (B.8) into Eq. (B.6) gives

$$
\begin{aligned}
\dot{J}_{\text {orb }, \text { MT }}= & \underbrace{J_{\text {orb }}\left[\frac{\dot{M}_{1, \text { ej }}^{\text {comp }}}{M}\left(\frac{1}{q}-\beta q\right)\right]+m \frac{a\left(1-e^{2}\right)}{1+e \cos v} \mathcal{T}}_{\dot{J}_{\text {orb RLOF }}} \\
& +\underbrace{J_{\text {orb }}\left(\frac{\dot{M}_{1, \text { loss }}}{M} \frac{1}{q}+\frac{\dot{M}_{2, \text { loss }}}{M} q\right)}_{\dot{J}_{\text {orb lost }}}
\end{aligned}
$$

where $q=M_{1} / M_{2}, \dot{J}_{\text {orb,RLOF }}$ is the torque acting on the orbit as a consequence of RLOF, while $\dot{J}_{\text {orb,lost }}<0$ is the torque applied 
by the material leaving the system. The corresponding torque applied onto the transferred mass is just

$\dot{J}_{\mathrm{MT}}=-\dot{J}_{\text {orb,MT}}$.

Using Eqs. (B.9) and (B.10) with $e=0$ gives Eq. (15).

Next, we demonstrate the consistency of Eq. (B.9) by showing that in the classical formalism for conservative mass transfer $\dot{J}_{\text {orb,RLOF }}=0$. If all material is transferred to the secondary $\left(\alpha_{\text {self }}=0, \beta=1\right)$, if the stars are treated as point masses $\left(r_{\mathcal{L}_{1}}=0\right.$, $\left.r_{\text {acc }}=0\right)$, and if we neglect the gravitational attraction by the accretion stream $\left(\boldsymbol{f}_{1}=0, \boldsymbol{f}_{2}=0\right)$, Eq. (10) reduces to

$\mathcal{T}_{\text {comp }}=-\frac{\dot{M}_{1, \mathrm{ej}}^{\text {comp }}}{M_{1}}\left(q V_{2, t}+V_{1, t}\right)$,

where $\dot{M}_{2, \text { acc }}=-\dot{M}_{1, \mathrm{ej}}^{\text {comp }}$ for conservative mass transfer. Sepinsky et al. (2007b) and Luk'yanov (2008) demonstrated that, if the orbital angular momentum is conserved, in a circular orbit $V_{1, t}$ and $V_{2, t}$ are related by

$q V_{2, t}+V_{1, t}=\left(\frac{G M}{a}\right)^{1 / 2}(1-q)$.

Substituting Eq. (B.12) into Eq. (B.11), and that result into Eq. (B.6) gives for a circular orbit

$$
\begin{aligned}
\dot{J}_{\text {orb }, \text { MT }}= & \dot{J}_{\text {orb,RLOF }}=J_{\text {orb }} \frac{\dot{M}_{1, \mathrm{ej}}^{\mathrm{comp}}}{M_{1}}\left(\frac{q}{1+q}\right)\left(\frac{1-q^{2}}{q}\right) \\
& -\frac{\dot{M}_{1, \mathrm{ej}}^{\mathrm{comp}}}{M_{1}} m(G M a)^{1 / 2}(1-q)
\end{aligned}
$$

where we have used $M_{1} / M=q /(1+q)$. Using Eq. (B.1), Eq. (B.13) reduces to zero, as required. 